The role of Focal adhesion Kinase in Flow induced signaling

\author{
Tobias Petzold \\ Potsdam, Germany
}

Physikum, Friedrich Schiller Universitaet Jena, Germany, 2004

A Thesis presented to the Graduate Faculty of the University of Virginia in Candidacy for the Degree of Master of Science in Biological and Physical Sciences

Department of Microbiology

University of Virginia

January 2007

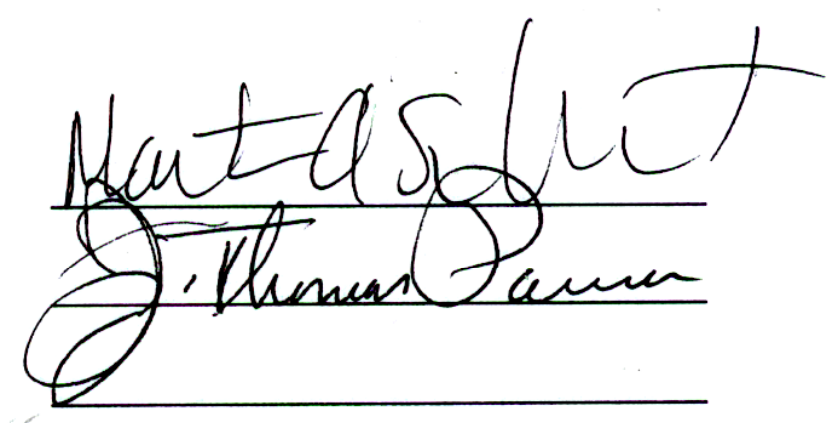




\title{
The Role of Focal adhesion kinase in shear induced signaling
}

\author{
Tobias Petzold
}

\begin{abstract}
:
Atherosclerosis is a chronic vascular inflammatory disease that develops in areas of disturbed flow like artery bifurcations and branch points. The transcription factor NF-kB is activated in atherosclerotic lesions and stimulates E-selectin, ICAM-1 and VCAM-1 expression, which mediate leukocyte recruitment. NF-kB activation by flow is downstream of integrin activation. Focal adhesion kinase (FAK) is a critical mediator of integrin signaling. To test FAK's role in flowinduced NF-kB activation, I isolated mouse aortic endothelial cells from conditional FAK knockout mice. Cre adenovirus caused complete loss of FAK from the conditional cells. Shear stress activated NF-kB in WT but not FAK-/endothelial cells, whereas Erk activation was similar in both cell types. While, reactive oxygen species (ROS) are critical mediators of flow induced NF-kB activation production their production was unaffected in FAK-/- cells, arguing the FAK might be involved in a distinct pathway of NF-kB activation by flow.
\end{abstract}




\section{Table of content}

Abstract $\quad$ i

Table of content $\quad$ ii

List of figures iv

List of abbreviations $\quad$ V

1 Introduction 1

1.1The vasculature 2

1.2Forces in the vasculature 2

1.2.1Pressure 2

1.2.2Shear stress 3

1.2.3Calculation of Shear forces 4

1.2.4Blood fluid dynamics 5

$\begin{array}{ll}\text { 1.3Flow conditions } & 6\end{array}$

1.4Flow mechanosensors in Endothelial cells 8

$\begin{array}{ll}\text { 1.5Integrins and flow induced signaling } & 10\end{array}$

1.6Focal adhesion kinase 12

$\begin{array}{ll}\text { 1.7Nuclear factor kappa B (NF-kB) } & 14\end{array}$

1.8Atherosclerosis and Flow $\quad 15$

$\begin{array}{ll}\text { 1.9Summary and hypotheses } & 16\end{array}$

\section{Materials and Methods}

2.1Mouse aortic endothelial cell isolation 18

$\begin{array}{ll}2.2 \text { Cell culture } & 18\end{array}$

2.3Virus transfection for immortalization and gene knock out 18 
2.4Cell characterization Dil-Ac LDL uptake assay 19

2.5Immunohistochemistry 19

2.6Immunoblotting 20

2.7Flow experiments 21

2.8Reactive oxygen species assay using Dichlorofluorescein-diacetate 22 (DCFH DA)

\section{Results}

3.1Mouse Aortic endothelial cells (MAEC)

3.3The Role of reactive oxygen species (ROS) in the activation of NF-kB by 32 flow

4 Discussion and future directions 


\section{$\underline{\text { List of Figures }}$}

Figure 1A Outsprouting primary endothelial cells day $11 / 18 \ldots \ldots \ldots .26$

Figure 1B Endothelial cell charaterisation by immunoblotting for VE-cadherin

Figure 1C Endothelial cell characterization by Dil-Ac LDL uptake $\cdots$

Figure 2A FAK-/- endothelial cell characterization by 27 Immunoblotting ......................................

Figure 2B FAK-/- endothelial cell characterization by 27 Immunofluorescence microscopy......................

Figure 3

Flow system scheme

21

Figure 4A Flow induced Erk phosphorylation time course

28

Figure 4B Flow induced p65 phosphorylation time course............

Figure 4C

Flow induced ICAM-1 expression

30

Figure 5A Flow induced production of reactive oxygen species ......

31

Figure 5B

ROS stimulated p65 Phosphorylation 


\section{$\underline{\text { List of abbreviations }}$}

\begin{tabular}{|c|c|}
\hline eNOS & Endothelial Nitric oxide synthase \\
\hline ERK & Extracellular regulated kinase \\
\hline GAP & GTPase activating protein \\
\hline $\mathrm{H}_{2} \mathrm{O}_{2}$ & Hydrogen peroxide \\
\hline ICAM -1 & Intercellular adhesion molecule-1 \\
\hline $\mathrm{IL} 1 / 6 / 8$ & Interleukin $1 / 6 / 8$ \\
\hline JNK & Jun N-terminal kinase \\
\hline LPS & Lipopolysaccharide \\
\hline MAPKKK & Mitogen activated protein kinase kinase kinase \\
\hline Mn-SOD & Manganese superoxide dismutase \\
\hline MRI & Magentic resonance imaging \\
\hline NO & Nitric oxide \\
\hline $\mathrm{Pa}$ & Pascal pressure unit \\
\hline PECAM-1 & Platelet endothelial cell adhesion molecule- 1 \\
\hline PI3K & Phosphoinositide-3 kinase \\
\hline PKA & Protein Kinase A \\
\hline SD & Standard deviation \\
\hline $\mathrm{SH} 2 / 3$ & Src homology domain $2 / 3$ \\
\hline SREBP & Sterol regulatory element binding protein \\
\hline $\mathrm{TNF} \alpha$ & Tumor necrosis factor $\alpha$ \\
\hline VE-cadherin & Vascular endothelial cadherin \\
\hline
\end{tabular}




\section{The role of Focal Adhesion Kinase in shear induced signaling}

\section{$\underline{1 . I n t r o d u c t i o n}$}

Cells can sense a wide range of environmentally and internally generated forces. Proper sensation, transmission and transduction into a regulated, physiological response by the cell is essential to prevent diseases. Subsequent diseases range from embryonic malformation, such as congenital deafness, to degenerative diseases like atherosclerosis or osteoporosis.

The establishment of left-right asymmetry in mammalian embryos is an essential step during embryogenesis. Accumulating evidence suggests a model in which motile cilia in the ventral node induce a leftward flow of extraembryonic liquid which activates polycystin-2, a cationic ion channel, associated with nonmotile cilia on the left side of the flow [1]. Ion channel activation triggers a $\mathrm{Ca}^{2+}$ influx, establishing a left- right gradient that induces more complex differentiation events. At the other end of the life span continuum, decreased mechanical stimulation results in the onset of osteoporosis. Besides complex hormonal regulation, life style changes disturb the tightly controlled equilibrium of bone production and decomposition. Osteocyte protrusions are surrounded by liquid in the lacuno-canalicular system of the unmineralized bone precursor matrix and are involved in the sensation of mechanical stimuli. Compression forces exerted during physical activity induce a liquid flow that causes shear stress (8-30 dynes/cm) on the cell surface of the osteocytes, which stimulates the production of metabolic factors like NO, prostaglandin 2 or insulin-like growth factor leading to bone formation through enhanced osteoblast function.

In the following, I will discuss shear stress induced mechanotransduction, and its potential role in the development of atherosclerosis as well as the potential role of focal adhesion kinase in mediating this process. 


\subsection{The vasculature}

The vasculature can be divided into the arterial high pressure system (big and small arteries, arterioles and precapillary vessels) and the venous low pressure system (postcapillary venules, veins and the right atrium) that supplies tissues between them with nutrients and clears metabolic waste.

Blood vessels except for the capillary tree tissues show a similar structure. The inner surface of the vessel is lined by a monolayer of endothelial cells which, under healthy conditions, is the only layer to interact with the flowing blood. A surrounding basal lamina completes the so called intima. The adjacent media represents the broadest layer and consists predominately of vascular smooth muscle cells. A thin fibrous adventitia covers and stabilizes the vessel and integrates it into the surrounding body tissue.

\subsection{Forces in the vasculature}

The different forces that predominate inside the vasculature are results of the cardiac cycle which drives a pulsatile blood flow through the vasculature and generates wall shear stress and cyclic strain which differently affects vessel function. Those are considered the two major forces.

\subsubsection{Pressure}

Blood pressure acts perpendicularly to the direction of flow on the vessel wall and is experienced by all components of the vessel. Periodic changes in circumferential stress and stretching forces due to rhythmical changes in blood pressure are necessary to maintain proper vessel structure and functionality, whereas chronic changes in transmural pressures can lead to vessel remodeling [4]. The relationship between wall thickness and circumferential stress $(\mathrm{T})$ in the wall fits into Laplace's law $T=P r / h$. Hence, increased transmural pressure (P; $\mathrm{r}$ represents the inner vessel radius), as found in hypertension, can be compensated by an increase of the wall diameter (h), 
thus keeping the circumferential stress $(\mathrm{T})$ inside the wall constant. The pathophysiological correlate of this relationship in hypertensive patients is hypertrophic remodeling of heart and vessels. Interestingly, circumferential stress remains similar between different organisms irrespective of their size, and seem to favor certain values[2].

\subsubsection{Shear stress}

The second, even much smaller force is wall shear stress. It is a frictional force exerted by the flowing blood on the endothelium. Thus, endothelial cells are the primary sensor for shear stress in the vasculature. The importance of shear force in the development of diseases such as atherosclerosis or vein graft disease prompted clinical investigations on in vivo measurements.

The assessment of shear forces in vivo is challenging, as direct flow measurements in humans require invasive catheterization of the patient that bears serious risks of procedural complications. Therefore, most flow measurements use indirect minimally invasive or noninvasive methods.

In arterioles, fluorescent labeled nanoparticles can be followed by video recordings to yield velocity and radial position profile inside a vessel that can be used for calculations. In larger and clinically more relevant vessels, ultrasound and MRI remain the method of choice. Both systems measure velocity profiles inside the vessel with the disadvantage that neither can access shear forces closer than $250-300$ um or 1,000-1,200 um respectively, to the vessel wall due to their physical resolution [3].

\subsubsection{Calculation of shear forces}

As calculations of shear force inside the vessel are challenging, physical fluid dynamic calculations are useful to determine blood flow derived shear forces, even though it uses a simplified model. Under hypothetical conditions, assuming a stiff tube and Newtonian fluid 
properties, an optimal parabolic flow velocity profile would develop, with centrally higher and peripherally slower flow speed.

Approaching flow profiles numerically by assuming that each liquid layer can be defined by a specific velocity and distance from the center (radius) leads to the definition of shear rate $(T r)$. Therefore, changes in velocity $(v)$ are related to the changes in the radius $(r)$.

(1) $\operatorname{Tr} \sim \frac{d v}{d r}$

The introduction of viscosity $(\eta)$ as constant in this relationship yields a Shear stress $(T)$ definition.

(2) $T=\eta \frac{d v}{d r}$

Derived from Poiseuille's law,

(2a) $T=\eta \frac{4 \mu Q}{\pi R^{3}}$

shear in vitro can be expressed as the product of

(3) $T=\frac{\eta 4 q}{\pi r^{3}}$

where $\eta$ is the viscosity, $q$ is the blood flow and $r$ is the lumen radius. In other words, $T$ is proportional to the blood flow and inversely proportional to the radius of the tube.

Integration of ultrasound or MRI (see above) recorded velocity profiles yields mean wall shear stress values which differ, depending on the localization inside the vasculature. A clinically relevant example is the human common carotid artery which is easily accessible for externally applied ultra sound. Doppler ultrasound of the carotid artery is commonly used to show turbulence in blood flow in the bifurcation area, helping to assess the risk of stroke or other progressions of atherosclerosis. 
In the common carotid artery, shear forces reach peaks of 2.6-4.3 $\mathrm{Pa}$ (depending on the study[3]; $0.1 \mathrm{~Pa}=1$ dynes $/ \mathrm{cm}^{2}$ ), with corresponding velocities of $62-105 \mathrm{~cm} / \mathrm{s}$ and mean shear stress values of 1.2-1.4 Pa.

The calculation of shear forces by integrating acquired flow profiles in fluid dynamic equations assume hypothetical experimental condition which are rather unphysiological. Inside the vasculature, blood flow conditions are more complicated due to the fact that blood is a nonNewtonian liquid, artery walls are elastic and that blood flow undergoes cyclic fluctuations.

\subsubsection{Blood fluid dynamics}

\section{Viscosity}

Blood is a non-Newtonian liquid that consists of a corpuscular fraction, comprised of erythrocytes, leucocytes, platelets and a plasma fraction that contains fibrinogen [82] and the different plasma protein fractions. Therefore, blood viscosity changes, depending on the flow environment. Heavy erythrocytes tend to travel in the center of the vessel whereas smaller platelets and plasma flow as a less viscose layer close to the endothelium and reduce the shear forces on the vessel wall (see equation 3) [3]. In contrast, under low shear, erythrocytes tend to form big aggregates mediated by plasma proteins like fibrinogen, $\alpha 2-$ macroglobulin and thus increase the apparent viscosity [83].

\section{Temporal fluctuation}

Additionally, blood flow and shear forces undergoes prominent cyclic fluctuations due to the cardiac cycle in vivo. During a short systolic output phase about $400 \mathrm{ml}$ of accelerated blood are released in the circulatory system reaching velocities up to $1.2 \mathrm{~m} / \mathrm{s}$ in the ascending aorta and subsequently causing higher wall shear stress values. During a much longer ( 2 thirds of a cycle) 
diastole the pressure and velocities decrease smoothly, supported by the energy reservoir function of the aortic arches (so called Windkessel function) yielding lower shear stress [83].

Taken together, the determination of shear stress values remains challenging. Besides technical restrictions of acquiring flow profiles close to the vessel wall, fluid mechanical calculations barely consider blood specific fluid properties. Hence, calculated shear forces represent approximations of in vivo values. This is important for designing flow experiments where stimulation with physiological shear forces is essential. For laminar flow studies on human and bovine endothelial cells, shear forces of 1.2 Pa seem reasonable and are close to calculated ones in the common carotid artery.

\section{$\underline{1.3 \text { Flow conditions }}$}

The calculations stated above address only the quantitative component of shear. Accumulating in vivo and recent in vitro studies (see later) show the importance of flow conditions, a qualitative component in flow induced signaling.

Three kinds of flow can be distinguished: laminar, oscillatory and turbulent flow. Laminar flow requires the development of a (nearly) parabolic flow profile, where differently fast liquid layers flow in parallel manner. Under physiological condition it yields high, unidirectional shear forces (averages between 15 to 20 dynes $/ \mathrm{cm}^{2}$ ), as found in large straight arteries in the vasculature [4]. High laminar, unidirectional shear stress induces an atheroprotective environment in the endothelium by maintaining an anti-inflammatory, anti-thrombotic, anti-apoptotic, and antihypertrophic state. [5]

Oscillatory and turbulent flow show significantly lower mean magnitudes in shear stress multidirectionality, and flow separation [4]. They are found in atheroprone regions in the vasculature like the outer walls of vessel bifurcations (e.g. carotid bifurcation), disposal dividers (e.g. Ostium A. renalis) or the lesser curvature of the vessel (aortic arch), and are thought to trigger endothelial 
dysfunction. Endothelial dysfunction is a pro-inflammatory, pro- thrombotic, and hyperthrophic activation state of the endothelium.

Oscillatory flow shows reversal of the direction of flow and secondary flow interference. Turbulent flow is a randomized motion of the blood, leading to vortices, eddies and other flow fluctuations that lack an ordered directionality and force. The transition from laminar to turbulent flow is marked by the dimensionless Reynold number. It integrates the ratio between inertial forces and viscous forces together with inner cross sectional diameter (ri), the mean velocity $(\vec{v})$, the density (p), and viscosity of the liquid $\left(\operatorname{Re}=2 \mathrm{ri} \vec{v} \frac{\rho}{\eta}\right)$. High Reynold numbers (e.g. $2.3 \times 10^{3}-$ $4 \times 10^{4}$ ) mark the transition from laminar to turbulent.

Using a computer controlled cone and plate viscometer, Gimbrone Jr. et. al. [6] were able to reproduce MRI acquired shear force waveforms from atheroprotective (common carotid) and atheroprone (internal carotid sinus) areas. Endothelial cells do not align in response to atheroprone flow, show decreased expression of junctional proteins like connexin 37 and show sustained NF-kB activation and inflammatory gene expression. Interestingly, in vivo data were interpreted to suggest that low flow might be clinically more relevant than disturbed flow. Cheng et al [7] implanted casts surrounding the carotid arteries to yield areas of low (upstream of the cast) and oscillatory flow (downstream). While rupture-prone vulnerable plaques with higher inflammatory gene expression (i.e. ICAM-1, VCAM-1, IL-6) developed on low flow sites, stable plaques were localized to oscillatory flow areas. Importantly, cast implantation changes not only flow conditions, but also pressures exerted on the vessel. Hence, it might be likely that both increased pressure and low shear forces can trigger the formation of vulnerable plaques.

Considering the above mentioned data, it is crucial to carefully distinguish between high and low shear stress (quantitatively) on the one hand and laminar and disturbed flow on the other (qualitatively) None the less, it seems that cells react in a similar way toward laminar and 
disturbed flow by using the same signaling pathways. Evidence for this comes from the observation that signaling pathways such as NF-kB, which are transiently activated by laminar flow, show a sustained activation under disturbed flow. This argues that continual changes of the direction of flow trigger sustained activation and prevents the downregulation of these pathways as found under laminar flow conditions[1]. Hence, an understanding of signaling pathways which are activated by laminar flow might allow the analysis of pathways that lead to the development of atherosclerosis in areas of disturbed flow. Another conclusion can be made that cells might use the same mechanosensors and transduction pathways to induce physiological or pathological signaling pathways. Thus, further elucidation of new mechanosensors will be very important.

\subsection{Flow mechanosensors in endothelial cells}

Besides the fact that so far, only one shear mechanosensor has been unambiguously identified (see later), an earlier hypothesis claimed a decentralized mechanotransduction model in which luminal shear forces can be sensed by different mechanosensors. In this model, mechanical forces are transmitted through the actin cytoskeleton to force-resistant anchor sites such as cell-cell junctions, focal adhesions or the nuclear membrane [8]. The importance of the cytoskeleton can be easily observed by endothelial cell alignment parallel to the direction of flow after prolonged stimulation (at least 12-16h). Cell alignment reflects the formation of parallel actin stress fibers, which enhance rigidity underneath the membrane, and by the reorientation of the microtubule organization center (MTOC) in vitro [9] and planar cell polarity in vivo[10]. Shear stress experienced by the cell is a function of cell shape and geometry and micro geometry changes (like alignment) can decrease average shear forces by $40 \%$, thereby modulating signaling thresholds [8].

Ion channels are activated by onset of shear within seconds which triggers $\mathrm{Ca}^{2+}$ and $\mathrm{K}^{+}$influx into the cell [11]. Subsequently, slight membrane potential shifts toward hyperpolarisation can 
feed forward to current-sensitive ion channels that can induce massive ion redistributions. This triggers cellular signals, since ions such as $\mathrm{Ca}^{2+}$ function as second messengers in the induction of eNOS and protein kinase $\mathrm{C}(\mathrm{PKC})$ activity. It remains unclear if ion channels are activated directly by shear forces or indirectly by flow induced intracellular signals.

The cell bilayer membrane itself may be shear responsive, as it shows an increase in fluidity [12] on the upstream site of the cell [13] after onset of flow. Furthermore, membrane fluidity can affect flow induced activation of mitogen activated protein kinases (MAPK), ERK, and JNK. The addition of benzyl alcohol increases membrane fluidity as assessed by the diffusion coefficient for Dil dye and enhances ERK and JNK activaton by flow. By contrast, cholesterol addition decreases membrane fluidity and MAPK activation by shear [14].

Other data suggests that the glycocalyx, a $50-400 \mathrm{~nm}$ thick polysaccharide matrix, on the extracellular side of the membrane, can transmit shear forces. Disturbance of the glycocalyx by partially digesting its heparan sulfate component can abolish shear induced NO production [8]. Recently, the role of caveolin in mechanotransduction was confirmed in vivo. Caveolae are sphingolipid and cholesterol rich membrane invaginations that contain caveolin-1 (cav-1) as an essential component. Cav-1 knock out mice showed disturbed short (i.e. reduced eNOS activation) and long term (i.e. increased intima proliferation ) adaptation toward flow, compared to endothelial cav-1 rescued control mice[15]. Of note, Cav-1 is an endogenous inhibitor of eNOS. Hence, eNOS activation by flow might vanish due to higher basal activity. The importance of cell-cell interactions in mechanosensation was underlined by Tzima et al [16]. They pointed at a mechanosensory complex that is located at the cell-cell junction. Whereby PECAM-1, an immunoglobulin family member, acts as direct mechanosensor in a functional complex with the junctional adhesion molecule VE-cadherin, and the VEGF receptor tyrosine kinase VEGFR2. Activation of this complex leads to PI3K mediated activation of integrins. 
Furthermore, disruption of this complex, as found in corresponding knockout mice, decreases the activation of NF-kB in atheroprone areas in vivo.

Integrins are major players in mechanotransduction, as their contact sites, such as focal adhesions and focal complexes, are regulated by mechanical forces generated by actin-myosin contractility and matrix rigidity. Integrin adhesions provide stable anchor points for external applied physical forces like strain-stretch or shear forces [17] and influence many different downstream signaling events.

\subsection{Integrins and flow induced signaling}

Integrins are a family of cell surface receptor proteins that mediate cell attachment mostly to extracellular matrix (ECM) proteins, like fibronectin, vitronectin, collagen, and laminin [18]. Integrin receptors are heterodimers of one of $18 \alpha$ and one of 8 know $\beta$ subunits, which form more than 20 known noncovalently associated receptor pairs. In that process, different combinations of integrin subunits lead to ligand specificity.

Integrin signaling regulates multiple cellular processes including cell migration, proliferation [19], apoptosis, tumor growth, tumor angiogenesis, metastasis [20] or mechanotransduction [17]. New integrin ECM interactions are sometimes required for downstream signaling in flow. In this process, intracellular signals induce talin binding to the inactive $\beta$ integrin subunit, disrupts an intermolecular saltbridge between the cytoplasmic tails, triggering conformational changes in the extracellular domain, thereby increasing integrin binding towards their ECM ligands [21, 22]. Integrin-ECM interactions are mediated by certain binding motifs such as the RDG (ArginineGlycine- Asparagine) motif, first discovered in fibronectin.

Although the short (50 amino acids or less except 34 ) cytoplasmic domains of both subunits show no intrinsic enzymatic activities, they contain specific phosphorylation/interaction sites for intracellular proteins. Liu [23] organized the binding partners into three groups: Actin-binding 
proteins [22] such as talin, involved in the integrin-cytoskeleton linkage and integrin clustering, signaling proteins such as FAK (although evidence for direct interaction is weak) and other proteins.

Integrin activation by flow is at least partially downstream of a junctional mechanosensor (see above). New integrin ECM interaction induces downstream signaling like Shc mediated MAPK activation. Preventing new receptor matrix engagement of avß3 integrins, using LM609 blocking antibodies, reduces the activation of MAPK[24]. Furthermore, integrin activation and ligation by flow modulates the activity of RhoGTPases like RhoA, Rac Cdc42, that are involved in cytoskeleton reorganization, RhoA-dependent SREBP activation, NF-kB activation and polarity determination by relocating the MTOC [25-28]. In addition, laminar shear stress over $18 \mathrm{~h}$ can upregulate the expression of $\alpha 5$ and $\beta 1$ integrins that lead to increased ERK activation and enforced anti- apoptotic signaling[29].

Orr et al [30] showed that NF-kB activation by flow is matrix- and consequently integrindependent. Cells plated on fibronectin, a binding partner for $\alpha 5 \beta 1$, or fibrinogen, an $\alpha v \beta 3$ binding partner, activate NF-kB, whereas collagen inhibits NF-kB via a p38 (MAPK) regulated pathway [30]. More recently, our lab showed that flow induced matrix specific integrin interaction can feed forward to inhibit talin-mediated integrin activation of matrix unspecific integrins. Following activation, FN binding $\alpha 5 \beta 1$ integrins stimulate $\mathrm{PKC} \alpha$, which inhibits $\alpha 2 \beta 1$ integrins interaction with collagen. In contrast, $\alpha 2 \beta 1$ suppresses $\alpha 5 \beta 1$ activation in a PKA dependent manner [31]. Besides regulating a variety of fundamental cellular responses as stated above, flow activated integrins can recruit downstream signaling proteins like focal adhesion kinase (FAK ) or p130cas $[17,24]$. In my study I will address the role of FAK in flow induced signaling. FAK might, due to its recruitment to focal adhesion sites and its diverse signaling functions (see later), play a crucial role in shear induced integrin signaling pathways. 


\subsection{Focal adhesion kinase (FAK)}

FAK is a cytoplasmic non-receptor tyrosine kinase which is activated upon integrin-mediated adhesion and is localized to integrin adhesions [32]. FAK has a multi-domain structure that provides interaction sites with many different proteins. FAK is important in many dynamic morphogenetic processes including embryogenesis, angiogenesis, and metastasis (extensively reviewed [33-35]). In addition to adhesion, FAK is activated in response to growth factors, cellular stress, and mechanical forces. In the vasculature, FAK was reported to be essential for morphogenesis and development of blood vessels [36, 37]. FAK was shown to be involved in the resealing of adherent junctions after $\mathrm{H}_{2} \mathrm{O}_{2}$ induced gap formation measured by transendothelial resistance [38], and in endothelial barrier strengthening [39].

Analysis of FAK's functional significance in vivo is limited by the fact that a complete deletion leads to embryonic lethality in mice around day 8.5 [40]. Even endothelial cell-specific FAK knockout is lethal between embryonic day 10 and 11.5 due to vascular defects [36]. In vitro data using embryo derived mouse endothelial cells show that depletion of FAK disturbs the organization of fibronectin. Additionally, the establishment of parallel actin stress fibers throughout the cytoskeleton is reduced, favoring a circular accumulation of actin fibers around the cell periphery [41]. The regulation of Rho-GTPase by FAK -/- cells was shown to be important for focal adhesion turnover [42]. FAK-/- show increased Rho activity, likely mediated through an improper activation of a Rho GAP (e.g.p190RhoGAP) into focal adhesions of FAK /-cells, yielding a rounded compact phenotype. Taking all the data into account, it seems likely that FAK is involved in the migration and morphogenesis of endothelial cells during embryonic vasculature development.

While the complete mechanism of FAK activation is unknown, it is well accepted that integrin ligation and clustering stimulates FAK recruitment and subsequent FAK phosphorylation at the autophosphorylation site Tyr397 within the kinase domain. Phosphorylated Tyr397 can serve as a 
docking site for SH2 domains of proteins such as Src, PI 3-kinase and Nck-2. Src is proposed to phosphorylate other sites within FAK, such as Tyr576 and 577 in the kinase domain, as well as Tyr861 and Tyr925 in the C-terminus. Both Tyr 397 and Tyr925 can bind the adaptor protein Grb2 which activates the MAPK pathway through the SOS- Ras- Raf pathway. The FAK Cterminus contains proline-rich sequences capable of binding to SH3 domains in proteins including p130Cas and ASAP-1. The extreme C-terminus contains paxillin and talin-binding sites which serve to anchor FAK within the adhesion complex. FAK signaling can stimulate paxillin and p130Cas phosphorylation, both of which can recruit the adaptor protein Crk. Subsequent recruitment of the Dock180/Elmo- GEF complex by Crk activates the small GTPase Rac, which is implicated in the integrin-induced activation of both JNK and NF- $\kappa$ B. FAK dependent phosphorylation of paxillin induces recruitment of Dock180/ELMO. Paxillin also recruits COOL/PIX another Rac/Cdc42 GEF into adhesion sites [35], though this interaction is phosphorylation independent.

Shear stress induced integrin conversion into a high affinity state results in the formation of new integrin- matrix interactions [26] and tyrosine phosphorylation of FAK [24]. FAK signaling is involved in sterol regulatory element binding protein-1 (SREBP-1) activation [28]. Following onset of flow, FAK moves from large stable focal adhesions to smaller, more transient focal complexes in the lamellipodia, which is thought to mediate cell migration in response to flow. FAK inhibition in vivo, through liposome-mediated incorporation of a FAK Y397 antibody, inhibited flow-induced eNOS phosphorylation and vasodilation [43].

In summary, there is evidence that FAK is involved in shear induced signaling at least partially downstream of integrins. Shear activated integrin signaling was shown to regulate additional pathways where the role of FAK remains undetermined. Rac activation by flow is downstream of integrins and it is tempting to argue that FAKs ability to recruit a Rac GEF (as described above) is required for this. Along this line, Rac activation is required to activate NF-kB, a major player in 
the development of atherosclerosis. Hence, a potential role of FAK in Rac mediated NF-kB activation and in the development of atherosclerosis remains to be investigated.

\section{$\underline{1.7 \text { Nuclear Factor kappa B }}$}

$\mathrm{NF}-\kappa \mathrm{B}$ refers to a family of dimeric transcription factors, which regulate the transcription of multiple proteins such as growth factors, antiapoptotic proteins of the Bcl family and components of immune and inflammatory systems (reviewed in [44]). The best characterized NF- $\kappa \mathrm{B}$ family member is RelA, a dimer of p65 and p50 subunits (subsequently referred to as NF- $\kappa B$ ). NF- $\kappa$ B is activated by many stimuli including cytokines (e.g. TNF $\alpha$, IL-1), bacterial derived LPS, oxidative stress or shear stress [44]. NF- $\kappa \mathrm{B}$ is normally maintained in an inactive state through interactions with I $\mathrm{B}$ proteins, which sequester $\mathrm{NF}-\kappa \mathrm{B}$ in the cytosol by masking the nuclear translocation

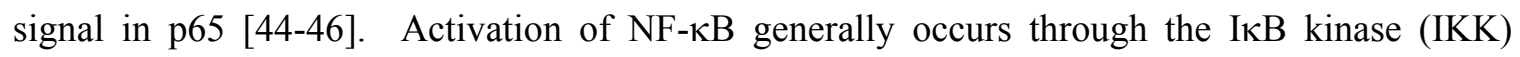
complex, consisting of 2 kinases (IKK $\alpha$ and $\operatorname{IKK} \beta$ ) and the regulatory subunit IKK $\gamma$ (a.k.a. NEMO). The activated complex phosphorylates $\mathrm{I} \kappa \mathrm{B}$, resulting in its ubiquitination and proteosomal degradation. The free $\mathrm{NF}-\kappa \mathrm{B}$ dimer translocates into the nucleus and binds to $\kappa \mathrm{B}$ enhancer sites where it recruits other cofactors and members of the transcription machinery inducing expression of target genes.

$\mathrm{NF}-\kappa \mathrm{B}$ activation is thought to play a major role in the pathogenesis of atherosclerosis [45]. Stimulating endothelial cells with shear stress in vitro activates NF- $\kappa \mathrm{B}$, which binds to the shear stress responsive element (SSRE) in target genes like ICAM-1, VCAM-1, E-selectins and chemokines like IL8 or monocyte chemoattractant protein. Expression of these proteins increase the adhesiveness of the endothelium toward leucocytes and further guides monocytes by diapedesis to the subendothelial space - triggering an inflammatory response [47]. NF- $\kappa \mathrm{B}$ subunits are elevated and NF- $\mathrm{BB}$ activation is observed in areas of disturbed flow in vivo 
concomitant with early expression of ICAM-1 and VCAM-1 [48]. These data suggest that NF$\kappa \mathrm{B}$ activation may be involved in the early stages of atherogenesis [45].

However, the exact mechanism of NF-kB activation by flow remains unsolved.

Previous work described a mechanosensory complex at cell-cell junctions (see above), which mediates shear stress stimulation of endothelial cells, leading to the conversion of integrins to a high affinity conformation [49]. High affinity integrins bind to the subendothelial matrix, and these newly bound integrins activate the Rac GTPase which is upstream of NF- $\kappa \mathrm{B}$ activation [27]. Active Rac by itself is an essential part of the NADPH complex which produces reactive oxygen species (ROS) [50]. While no redox-sensing component in the NF- $\kappa \mathrm{B}$ activation cascades is currently described, ROS are well established inducers of NF- $\kappa \mathrm{B}$ activation [51] and increased in atherosclerotic areas .

This evidence suggests into a hypothetical model that FAK is required to activate Rac by flow, which subsequently induces the production of ROS, triggers NF-kB activation and supports the development of atherosclerosis.

\section{$\underline{1.8 \text { Atherosclerosis and Flow }}$}

Atherosclerosis, a chronic inflammatory reaction within the vessel wall, is the major cause of death in Western nations, and showing increasing prominence in developing countries [52]. In fact, the development of atheroslcerosis is already determined during the anatomical formation of the vasculature system as an impressive study on Japanese children confirmed the early formation of fatty streaks, even in one year olds [53].

The pathophysiology of atherosclerosis is multi-faceted. Well defined systemic risk factors like hypertension, diabetes or dyslipidemia are experienced throughout the circulatory system. However, atherosclerotic lesions develop predominantely in areas of oscillatory, disturbed and generally low shear stress and show similar distribution in different species [5, 54, 55]. 
In atheroprone areas, low, oscillatory or disturbed flow leads to endothelial cell dysfunction accompanied by the activation of proinflammatory pathways [56-60] and changes of structural and functional integrity of the endothelium (see above).

Endothelial dysfunction favors an atheroprone, pro-thrombotic state, with proinflammatory pathways like NF-kB upregulated [45], while atheroprotective pathways, like NO production, and ROS scavenger expression, like Mn-SOD [61], are downregulated. In contrast, NADPH oxidase -dependent ROS production is increased in areas of atheroprone flow. A functional shutdown of NADPH oxidase enzymatic activity, as found in NADPH-p47 subunit knockout mice, reduces atherosclerosis [62].

In regions of disturbed flow, the barrier integrity of the endothelium is decreased. Chien et. al [14] showed that in atheroprone flow regions the endothelial cell turnover is higher and allows a transient increase in permeability for serum constituents like albumin and LDL, resulting in lipid accumulation in the intima [14]. Our lab described a different and/or additional shear induced mechanism mediated by p21 activated kinase (PAK). PAK activates MLC (Myosin light chain), thereby increasing the contractility of the cytoskeleton and inducing gap formation between neighboring EC [41]. Shear stress activates PAK in vitro and PAK is activated in areas of disturbed flow in vivo (Orr et al, unpublished). Low flow was shown to increase the permeability for Evans blue dye [63] in vivo. These data are consistent with increased cholesterol and modified lipoprotein accumulation in atherosclerotic lesion areas. In addition, the transcription factor NF$\mathrm{kB}$ is activated by flow and is thought to play a major role in the pathogenesis of atherosclerosis [45] (see above).

\subsection{Summary and hypothesis}

Understanding how flow stimulates signaling through FAK may help to elucidate new mechanotransduction pathways, to gain more detailed insights into how mechanosensory 
pathways work, and offer new therapeutic approaches to treat vascular diseases like atherosclerosis.

FAK is shown to be activated and recruited to integrins after shear stress stimulation. Its kinase activity and diverse binding domain structure reflects on its various functions in different cellular contexts. This combination of spatial accumulation at adhesion site and functional properties defines focal adhesion kinase as a potential player in shear force- induced signaling downstream of integrin activation by flow.

Shear stress activates Rac in an integrin-dependent manner which (likely) increases the production of ROS via NADPH oxidase. Following this idea, FAK might be required for Rac GTP loading as it can activate and recruit guanine nucleotide exchange factors such as DOCK180/Elmo or PIX into adhesion sites.

I hypothesize that FAK is a critical component of flow- and integrin-mediated activation of NF$\kappa \mathrm{B}$ by regulating the Rac-dependent production of reactive oxygen species in response to flow. Subsequently, FAK inhibition could attenuate the expression of NF-kB target genes which are involved in the development of atherosclerosis. 


\section{Material and Methods}

\subsection{Mouse Aortic Endothelial Cell (MAEC) Isolation}

All animal handling and procedures were approved by the animal care and use committee of the University of Virgina. We obtained 9 weeks old FAK conditional knockout mice (gift of Dr. Parsons, University of Virginia) and isolated mouse aortic endothelial cells (MAEC) by the aortic explant method as previously described [64]. In short, the isolated aorta was dissected into $1 \mathrm{~mm}$ long rings that were placed onto matrigel containing 6 well dishes. After 3-10 days, the EC started to migrate towards the bottom and form characteristic tube like structures. After 2 weeks, the tissue explants were removed. Upon reaching cell confluence, the matrigel was dissolved by adding dispase and cells were replated on $0.2 \%$ gelatin coated dishes. Selection for ECs was accomplished by replacing D - with L- valine in growth medium [65] supplemented with 15\% heat inactivated fetal bovine serum (FBS), $30 \mu \mathrm{g} / \mathrm{ml} \mathrm{ECGS} \mathrm{and} 50 \mu \mathrm{g} / \mathrm{ml}$ heparin.

\section{$\underline{2.2 \text { Cell culture }}$}

After passage 2, cells were maintained in Cambrex's EBM 2 endothelial cell medium containing $10 \% \mathrm{FBS}, 1 \%$ penicillin/streptomycin and $2 \mathrm{mM} \mathrm{L-} \mathrm{glutamine} \mathrm{(Invitrogen).} \mathrm{Cells} \mathrm{were} \mathrm{grown} \mathrm{to}$ confluence in plastic tissue culture dishes precoated with $0.2 \%$ gelatin

\subsection{Virus transfection for immortalization and Gene knock out}

Following passage 3 , cells at $70 \%$ confluency were immortalized with polyoma middle $\mathrm{T}$ virus to increase proliferation and survival. Briefly, $4 \mathrm{ml}$ of virus stock (unknown titer) containing $5 \mu 1 / \mathrm{ml}$ polybrene was added to cells and incubated for $16 \mathrm{~h}$. Cells were rinsed with phosphate buffered saline and fresh medium was added.

In passage 6, FAK flox/flox cells at $70 \%$ confluency were transfected with replication defective adenovirus containing a cre-recombinase GFP construct [66] (gift of Dr. Parsons, University of 
Virginia). The adenovirus was grown in HEK 293 cells and purified using adeno-X virus purification kit (Clontech). For transfection, $5 \mu \mathrm{l} / \mathrm{ml}$ of virus (unknown titer ) were added to culture dish medium and incubated for $20 \mathrm{~h}$. After 24 hours, the transfection efficiency was observed by GFP expression. Primary cell pictures in Figure 1A are courtesy of David Bolick (University of Virginia).

\subsection{Cell characterization by Dil-Ac LDL uptake assay}

Confluent cells were incubated with medium containing $5 \mu \mathrm{g} / \mathrm{ml}$ Dil-Ac-LDL. After $2 \mathrm{~h}$, the cells were observed by fluorescent microscopy (microscope described later, using a 550nm emission filter; 10x magnification) and screened for perinuclear accumulation of the dye. Cells were washed 3 times in PBS and further cultured.

\subsection{Immunocytochemistry}

One day before staining, cell were plated on $25 \mathrm{~mm}$ glass coverslips coated with $10 \mu \mathrm{g} / \mathrm{ml}$ fibronectin and blocked with $0.2 \%$ BSA. Coverslips were washed with ice- cold PBS, then fixed in $2 \%$ formaldehyde- PBS solution, washed with PBS, then permeabilized with $0.1 \%$ Triton-PBS for 15 minutes at room temperature (RT). To reduce nonspecific cross reactivity of the antibody, cells were incubated with $10 \%$ goat serum (GS) containing TBST $(0.05 \%$ Tween- 20$)$ for $1 \mathrm{~h}$ at RT. Primary antibodies: mouse anti- $\beta$ catenin (Santa Cruz Biotechnology) sc-7963, dilution 1:500, $2 \mathrm{~h}$ incubation ; mouse anti-FAK clone 4.47 (Upstate cell signaling), dilution 1:1000, 3h incubation ; mouse anti-paxillin (Zymed Lab.), dilution 1:500, 1h incubation; were diluted in blocking solution. After incubation with primary antibody, cells were washed and incubated with anti mouse IgG secondary -Alexa 568 (or 488) conjugated antibody for 1 h. Coverslips were washed PBS and mounted on the glass slide with Fluorochrome G. Images were acquired using a Nikon-DiaPhot microscope equipped with a Coolsnap video camera (ISEE software) with a 
10x or 60x oil immersion lens. Confocal Images were acquired using a Zeiss LSM 510 with 63x oil or 40x lenses. Images were processed with Adobe Photoshop 7.

\subsection{Immunoblotting}

Cells were directly lysed in $100 \mu$ l of SDS sample buffer (62.5 mM Tris-HCl (pH 6.8), 2\% w/v SDS, $10 \%$ glycerol, $50 \mathrm{mM}$ dithiothreitol, $0.1 \% \mathrm{w} / \mathrm{v}$ bromphenol blue). Using 10\% SDS-PAGE gel electroporesis (constant $100 \mathrm{~V}$ ), lysates were separated according to the molecular weight and transferred to PVDF membranes. Membranes were blocked with 5\% nonfat milk powder in TBST $(0.05 \%$ Tween) for $1 \mathrm{~h}$ and incubated with primary antibody: rabbit anti phospho- $\mathrm{p} 65$ (Cell signaling) dilution 1:1000; rabbit anti p65, (Santa cruz Biotechnology), dilution 1:1000; rabbit anti phospho-ERK, cell signaling, dilution 1:1000; rabbit anti ERK, (Cell signaling), dilution 1:1000; mouse anti Pyk2, (BD Bioscience) dilution 1:1000, overnight. Blots were washed with TBST and incubated with secondary anti rabbit or anti mouse IgG- horseradish peroxidase (HRP) conjugated antibodies (Jackson Immuno-research), dilution 1:5000. To visualize bands, Amersham Bioscience chemiluminescence reagent ECL and film were used. Generally, blots were first incubated with phospho-specific antibodies, then treated with re-blot plus stripping buffer and reprobed for total protein levels. Films were scanned and bands were quantified using Image G software. Phospho-specific bands were normalized to total protein levels. 


\subsection{Flow experiments}

Glass slides, 38x75mm (Corning), were extensively washed in $2 \mathrm{M} \mathrm{NaOH}$ in $70 \%$ ethanol, washed with water and ethanol, air dried and flame sterilized. A $0.5 \mathrm{~mm}$ thick gasket, that omits the flow chamber dimensions was placed on the glass slide which was coated with $10 \mu \mathrm{g} / \mathrm{ml}$ fibronectin in PBS, blocked with $0.2 \%$ BSA in PBS for $30 \mathrm{~min}$, and endothelial cells were plated overnight. To reduce cell baseline cell activation, cells were starved for $3 \mathrm{~h}$ in $0.2 \%$ FBS EBM-2 medium prior to all experiments. Our laminar flow system is designed to yield simple $2 \mathrm{D}$ parabolic flow profiles.

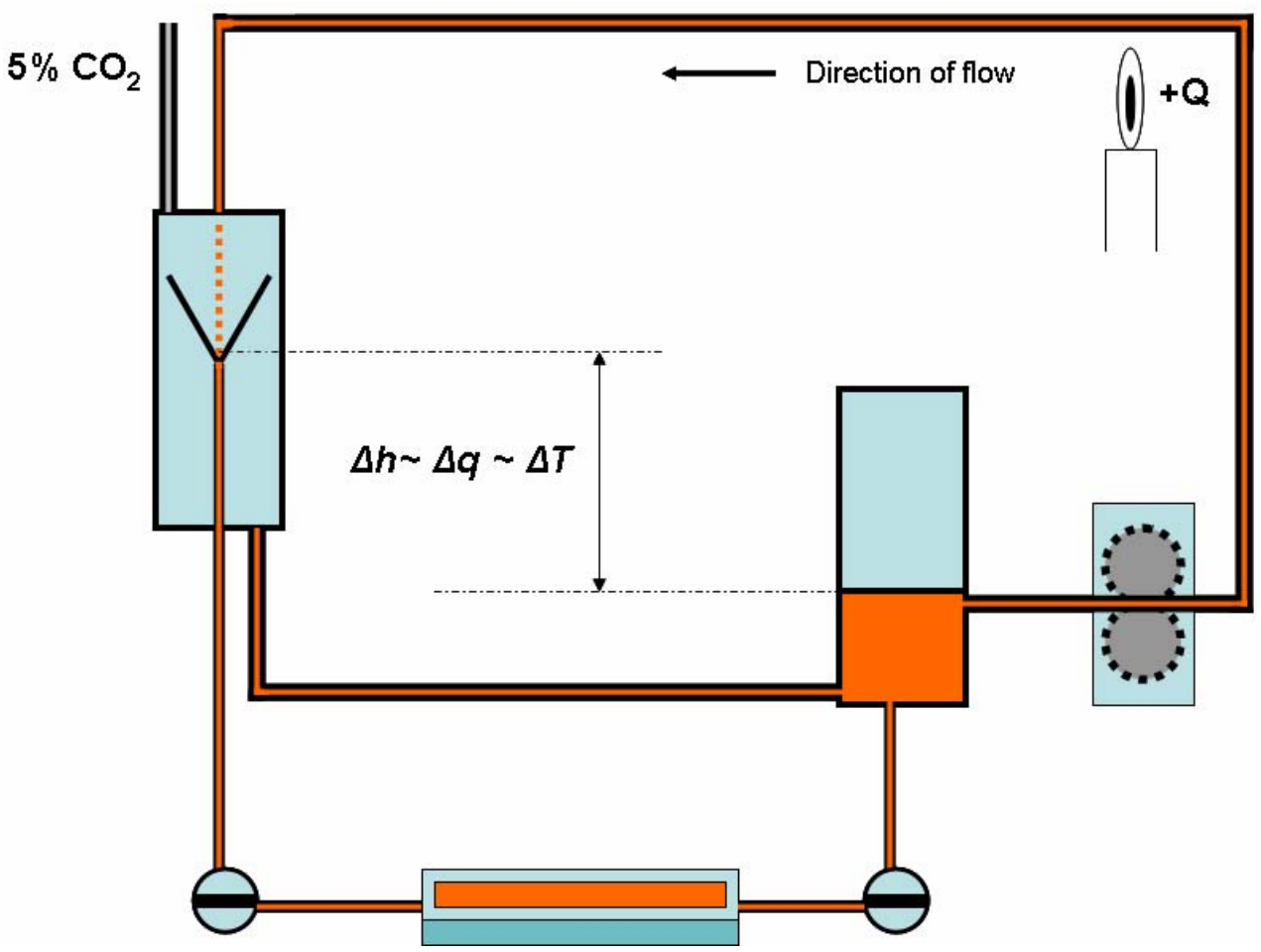

Figure 3: Flow system scheme. The height difference $\boldsymbol{\Delta h}$ between the reservoirs is proportional to the blood flow $\boldsymbol{q}$ and the shear forces $\boldsymbol{\Delta T}$. A roller pump returns the liquid back to the higher reservoir. A heat source $(+\mathrm{Q})$ and $\mathrm{CO}_{2}$ inflation keep Temperature and $\mathrm{pH}$ constant. The flow chamber is represented at the bottom. 
This flow profile is due to a short entrance length compared to total channel length and a much greater width than height $(b>>h)$ [67]. The driving force for flow is due to a hydrostatic pressure difference between the upper and lower reservoir, in our case it yields a 16 dynes $/ \mathrm{cm}^{2}$. The circulation is closed by a roller pump that returns the outflow medium back into the higher reservoir.

Temperature is kept constant at 37 degree Celsius by an external heating source while the $\mathrm{pH}$ is kept constant by introduction of a $95 \%$ air $/ 5 \% \mathrm{CO}_{2}$ mixture into the lower reservoir (Figure 3). Glass slides were placed and clamped onto the flow chamber and sheared in 0.2\% EBM2 medium for the indicated times.

\subsection{Reactive oxygen species assay using Dichlorofluorescein-diacetat (DCFH-DA)}

To quantify the amount of intracellular ROS, we used membrane permeable DCFH- DA[68]. DCFH is a redoxsensitive agent that after being oxidized to DCF by various oxidants, becomes fluorescent. Thus, fluorescence increases linearly with the amount of generated intracellular ROS. To allow the indicator uptake, cells were incubated in medium containing $10 \mu \mathrm{g} / \mathrm{ml}$ DCFH-DA for $30 \mathrm{~min}$ and then flowed in $0.2 \%$ FBS EBM2 medium, containing DCFH for $30 \mathrm{~min}$ at 16 dynes $/ \mathrm{cm}^{2}$. As a positive control, cells were kept under static conditions and stimulated with $0.6 \mu \mathrm{mol} \mathrm{H}_{2} \mathrm{O}_{2}$.

Cells were rinsed with PBS and cells were scraped into 1\% Triton- PBS, supplemented with $3 \mu \mathrm{l} / \mathrm{ml}$ BHT (butylated-hydroxyl-toluene) to prevent further shear-unrelated oxidation. Samples were alanlyzed in a 96 well plate reader using a $488 \mathrm{~nm}$ excitation and a 530-580nm emission filter set. Values were normalized to total protein amount using the Lowry protein assay[69] 


\section{$\underline{\text { 3. Results }}$}

\subsection{Mouse Aortic endothelial cells (MAEC)}

To determine the role of FAK in flow signaling I derived a polyclonal FAK flox/flox (conditional wild type) and a FAK -/- knockout mouse aortic endothelial cell line (MAEC). Since FAK knockout in mice results in embryonic lethality, Beggs et al [66] generated a conditional FAK knockout mouse using the Cre-loxP system targeting the second kinase domain. Subsequent Cre recombinase mediated excision abolished FAK protein expression [66]. I isolated and cultured aortas from FAK conditional knockout mice. At day 6 after aorta isolation, primary MAEC started to sprout (Figure 1A Day 11, 10x magnification) and formed a confluent monolayer at day 18. Next, primary cells were immortalized with polyoma middle $\mathrm{T}$ virus. The middle $\mathrm{T}$ antigen forms a multi- protein complex at the cell membrane with protein phosphatase2, src family kinases, phosphatidylinositol (3') kinase and ShcA. Middle T- antigen positively regulates the PI3K- PKB antiapoptotic pathway and the Src- ShcA- Ras mediated activation of mitogenic MAPK signaling [70].

As endothelial cells are highly differentiated, I tested if they retain this cell specific properties in culture. First, I observed the proper formation of a monolayer composed of cobblestone like endothelial cells (Figure 1A Day 18, 10x magnification). Next, I subjected whole cell lysates from FAK flox/flox cells, passage 3, and FAK -/- cells, passage 13, to immunoblotting for VEcadherin, a specific endothelial cell marker, which has crucial importance for shear signaling [16]. As shown in Figure 1B, MAEC express passage independent equal amounts of VEcadherin. Primary C57/B16 MAEC, passage 2, were used as positive control. HUVECS shows antibody specificity as the antibody was raised again human origin. SMC and 3T3 fibroblasts were used as negative controls. To identify potential contaminating cell populations, I tested for uptake of Dil- Ac- LDL. Endothelial cells express high levels of scavenger receptors on their surface and can internalize modified lipoprotein faster and accumulate it in a perinuclear location, 
compared to SMC or Fibroblasts. Therefore FAK flox/flox cells, passage 5, were incubated with $5 \mu \mathrm{g} / \mathrm{ml}$ Dil Ac -LDL containing medium for $2 \mathrm{~h}$ and observed under a fluorescence microscope using a red emission filter set. For quantification, 100 cells were counted and observed for perinuclear dye accumulation, I found that $98 \%$ of the cells showed the endothelial- specific staining pattern (Figure 1C). As a negative control, I used Mefs which were treated the same way but show cytosolic dispersed stain (not shown). To ablate focal adhesion kinase expression, I infected FAK flox/flox with a replication deficient adenovirus carrying a GFP Cre recombinase construct. The transfection efficiency was observed by fluorescent microscopy after $24 \mathrm{~h}$. Reproducibly, at least $80 \%$ of the cells show GFP and hence Cre recombinase expression (data not shown). After $72 \mathrm{~h}$ of incubation, cells were tested for remaining FAK expression by immunoblotting and immunocytochemistry. As shown in Figure 2A, gene excision abolishes detectable FAK protein expression. Figure 2 B shows that FAK localizes to adhesions, which form typical streak-like structures at the bottom side of FAK flox/flox but not FAK-/- cells. Pyk2, a FAK- related protein tyrosine kinase, is overexpressed in FAK-/- fibroblasts [71] and might compensate for the loss of FAK function. Therefore, I determined Pyk2 protein levels by immunoblotting. Both cell lines express similar amounts of Pyk2 (FAK flox/flox 1: 0.95 in FAK/- ) as seen in Figure 2A. As FAK is an important member of FA, I was curious if FAK-/- cells are still able to form proper FA. I stained for paxillin, a FA marker, which is independent of FAK recruited into focal adhesions [72] and couldn't observe any difference by fluorescent microscopy.

As the integrity of the endothelial monolayer is essential for its shear-sensitivity, we stained for ßcatenin, a cytoplasmic binding partner of cadherin receptors found in intercellular adherens junction. As shown in Figure 2B, both cell lines showed an accumulation of B-catenin at the cell border to neighboring cells. Taken together, I was able to derive conditional FAK flox/flox and 
FAK -/- knockout MAEC cell lines which retain their endothelial cell-specific characteristics in culture.

Figure 1: Cell characterization. A) Pictures show cells growing in Matrigel after aorta explantation. At Day 11 cells start to sprout and reach confluency on day 18. Pictures are courtesy of David Bolick, University of Virginia B) Whole cell lysates were subjected to immunoblotting for VE-cadherin. VE-cadherin protein levels remain similar in MAEC, passage independent. The higher signal in HUVECS is most likely because the antibody was raised against human protein C) To screen for potential contaminating cell populations FAK fl/fl p5 were incubated with $5 \mu \mathrm{g} / \mathrm{ml}$ Dil- AcLDL for $2 \mathrm{~h} .100$ cells were counted and observed for specific accumulation of the Dye, $98 \%$ of the cells show perinuclear dye accumulation. Pictures were acquired using Nikon-DiaPhot microscope with 10x lens with a red emission filter set, picture is miscolored.

Figure 2: FAK-/- MAEC characterization. A) FAK flox/flox and FAK-/- whole cell lysates were subjected to immunoblotting for FAK and Pyk2 and normalized to total Erk protein levels. FAK-/- cells show no detectable FAK protein expression. Pyk 2 expression levels are similar between both cell lines. B) Both cell lines were stained for FAK, paxillin and ß-catenin. FAK-/- cell lack FAK specific staining pattern as found in FAK fl/fl cells. Both cell lines show similar paxillin distribution in adhesion structures and $\beta$-catenin accumulation in intercellular junction inside the monolayer. Pictures were acquired using a Zeiss LSM 510 with 63x oil or 40x lenses. Scale bares represent $20 \mu \mathrm{m}(63 \mathrm{x})$ or $50 \mu \mathrm{m}$ respectively. 
Figure 1

A)

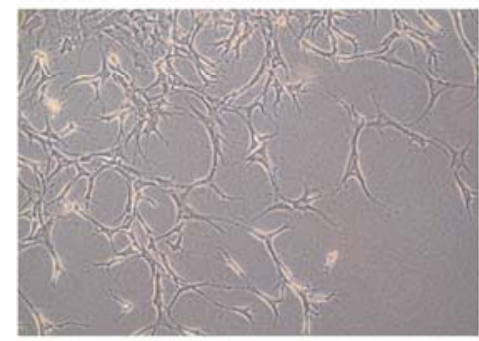

Day 11: migrating MAEC

(10x)

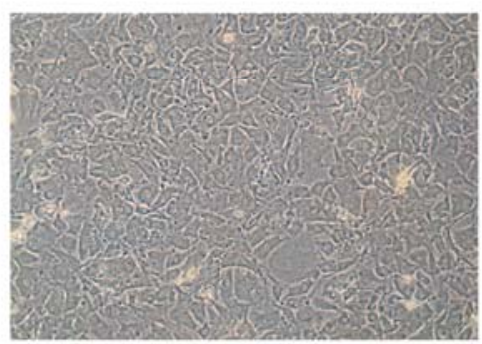

Day18: cobbelstone monolayer (10x)

B)

IB: Ve-cadherin

IB: Tubulin
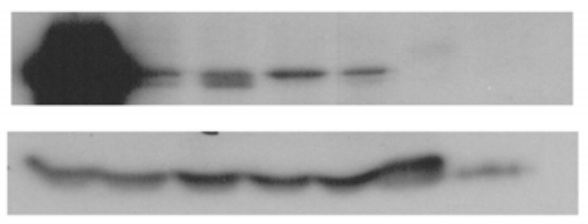

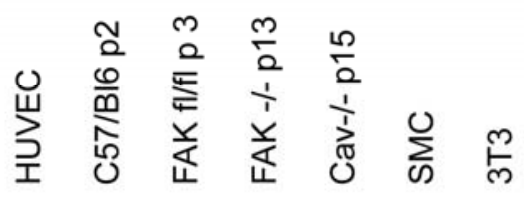

C)

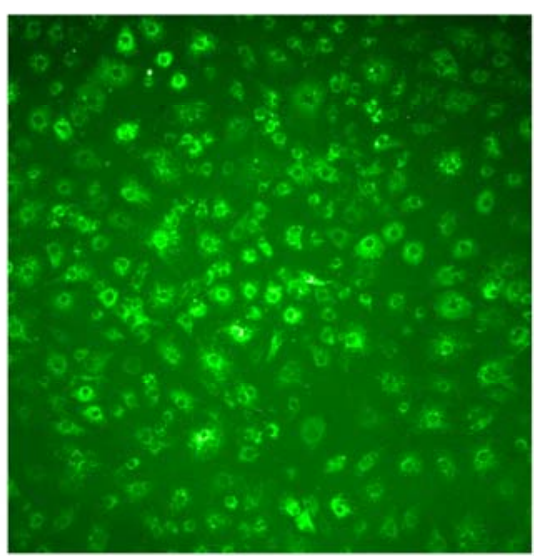

FAK fl/fl cells $p 5$ after $2 \mathrm{~h}$ DiL-Ac LDL treatment (10x).

$98 \%$ of cells show endothelial cell specific peri-nuclear dye accumulation . 
Figure 2

A)

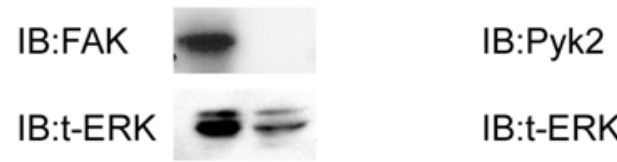

(1) $(0.95)$

MAEC FAK : fl/fl $\quad-/-$

B)

FAK

(63x)

Paxillin

(63x)
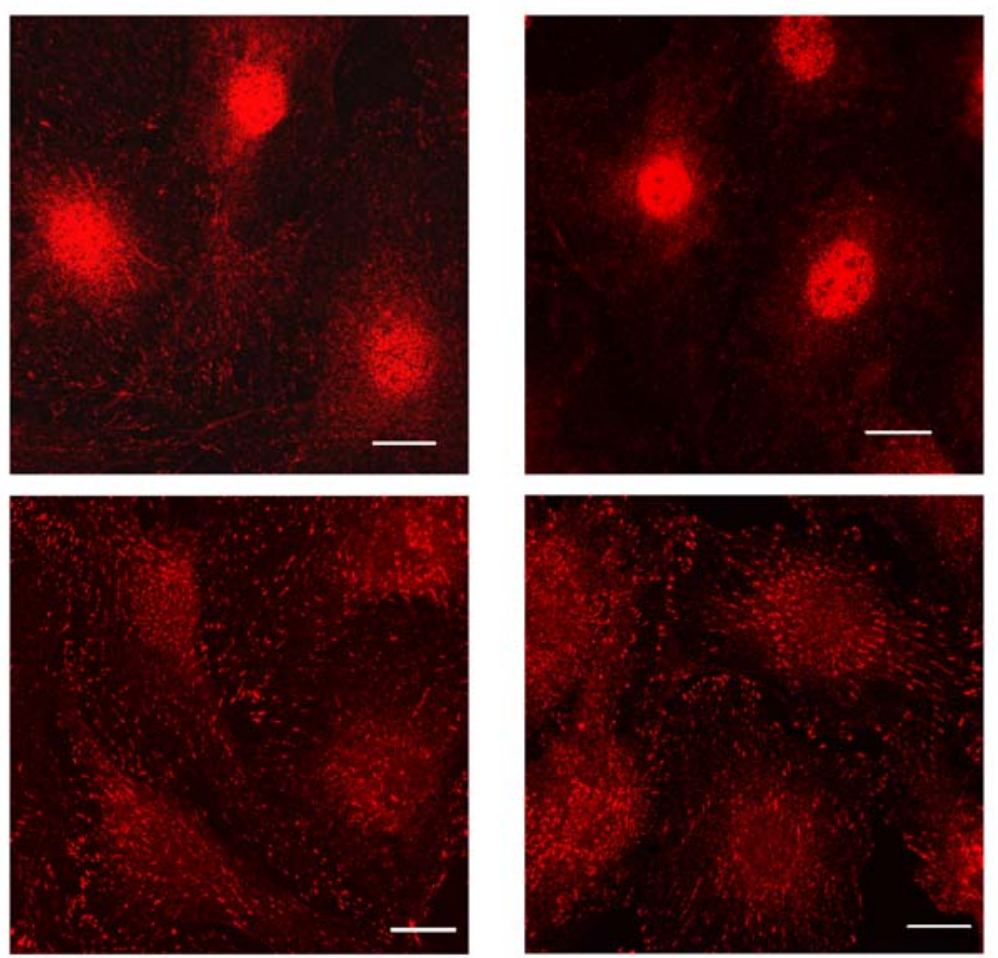

ß-catenin (40x)
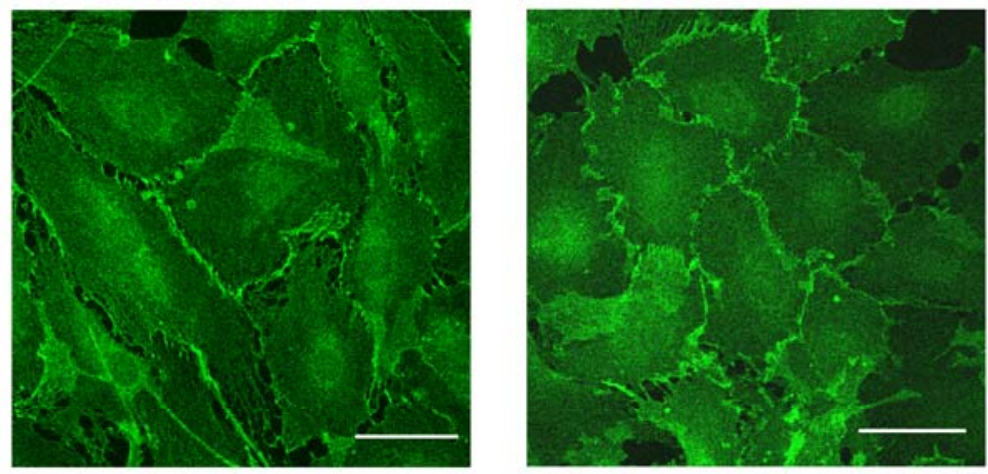

MAEC:

$\mathrm{fl} / \mathrm{fl}$

$-/-$ 


\subsection{Role of FAK in flow activation of $E R K$ and $N F-k B$}

To address the role of FAK in flow signaling we were interested in ERK and NF-kB activation. Therefore, FAK flox/flox control and FAK-/- cell were exposed to 16 dynes $/ \mathrm{cm}^{2}$ shear forces for $5,15,30$, and 60 minutes. The functional activation of ERK requires phosphorylation of Thr202 and Tyr204. We used a phospho-specific antibody to detect the phosphorylation state of both sites. As shown in figure 4A, both cell lines show a similar pattern of ERK phosphorylation after shear time, with a significant phosphorylation peak at $5 \mathrm{~min}$.

\section{IB: pErk \\ IB: t-Erk}

Shear

Time (min)

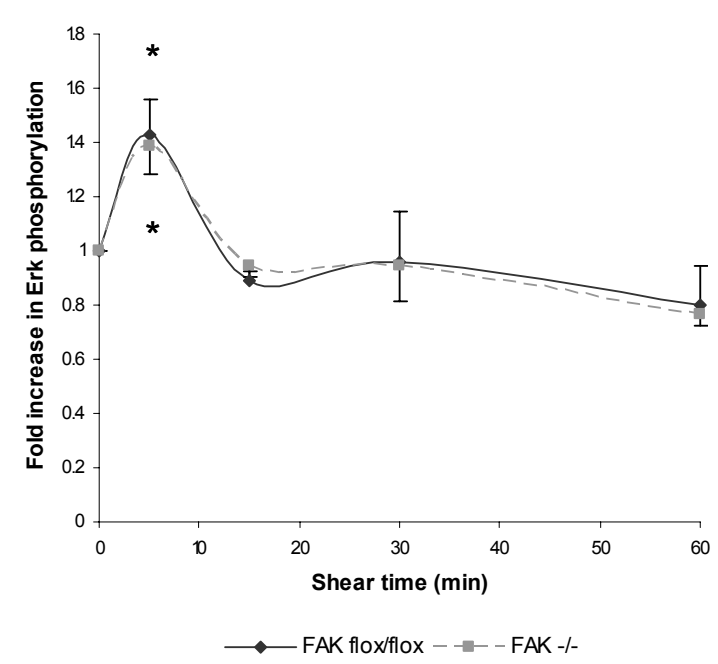

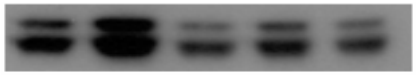

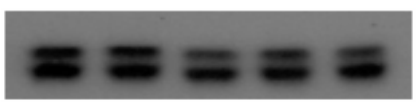

$\begin{array}{lllll}0 & 5 & 15 & 30 & 60\end{array}$

FAK- $/-$

Figure 4A: Flow induced Erk phosphorylation. Cell were sheared (16 dynes $/ \mathrm{cm}^{2}$ ) for the indicated times and subjected to immunoblotting for phosphoErk $\mathrm{N}=3$, Bars over time points represent standard errors. Asterisk represent significance towards time point $0, * \mathrm{P}=0.05$, Values are normalized to total ERK protein levels. 
IB: p65p

IB: t-Erk

Shear

Time (min)
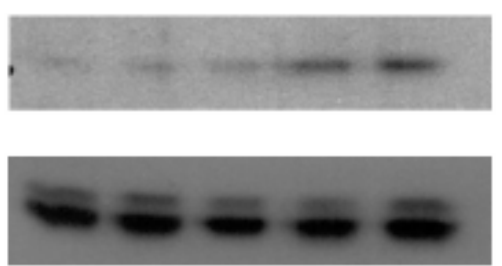

$\begin{array}{lllll}0 & 5 & 15 & 30 & 60\end{array}$

FAK flox/flox

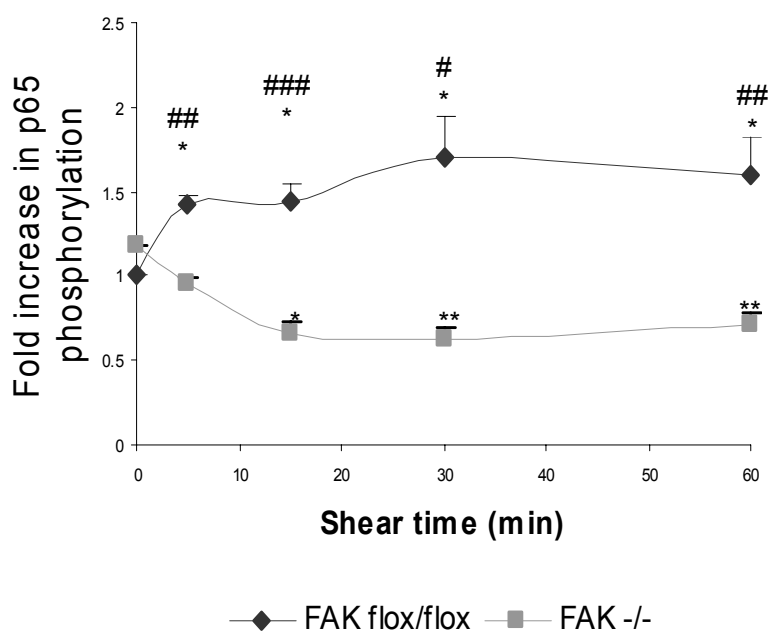

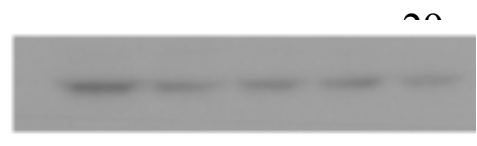

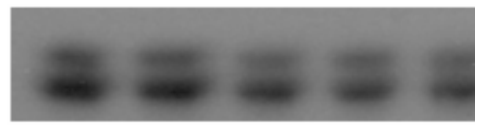

$\begin{array}{lllll}0 & 5 & 15 & 30 & 60\end{array}$ FAK-/-

Figure 4B: Flow induced p65 phosphorylation. Cell were sheared (16 dynes $\left./ \mathrm{cm}^{2}\right)$ for the indicated times and subjected to immunoblotting for phospho-p65 $\mathrm{N}=6$, Bars over time points represent standard errors. Asterisk represent significance towards time point $0, * \mathrm{P}=0.05$ ** $\mathrm{P}=0.01 . \quad$ Crosses represent significance between cell lines \# $\mathrm{P}=0.05 \quad \# \quad \mathrm{P}=0.01 \quad \# \# \# \quad \mathrm{P}=0.001$ Values are normalized to total ERK or p65 protein levels.

NF-kB is also activated by flow, which subsequently induces target gene expression, such as ICAM-1. Onset of flow triggers nuclear translocation and serine phosphorylation of NF-kB, both required for its transcriptional activity. I therefore assayed phosphorylation at Ser536 (figure 4B). In FAK flox/flox cells, p65 phosphorylation appears within the first 5 minutes and peaks at 30 min, consistent with published results [30]. Surprisingly, FAK -/- cells showed no increase in p65 phosphorylation, rather reaching a minimum after 30min. To address the physiological relevance of decreased p65 activity, we measured ICAM-1 protein expression levels. 
IB: ICAM-1

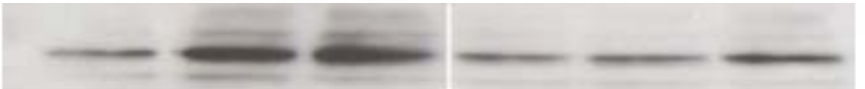

IB: $\mathbf{t - E r k}$

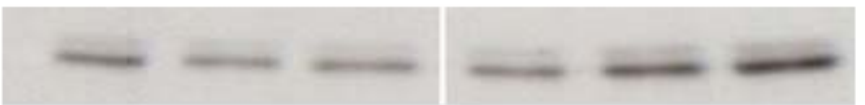

Shear

Time (h)

$\begin{array}{lll}0 & 1.5 & 3\end{array}$

FAK flox/flox $\begin{array}{lll}0 & 1.5 & 3\end{array}$

FAK-1-

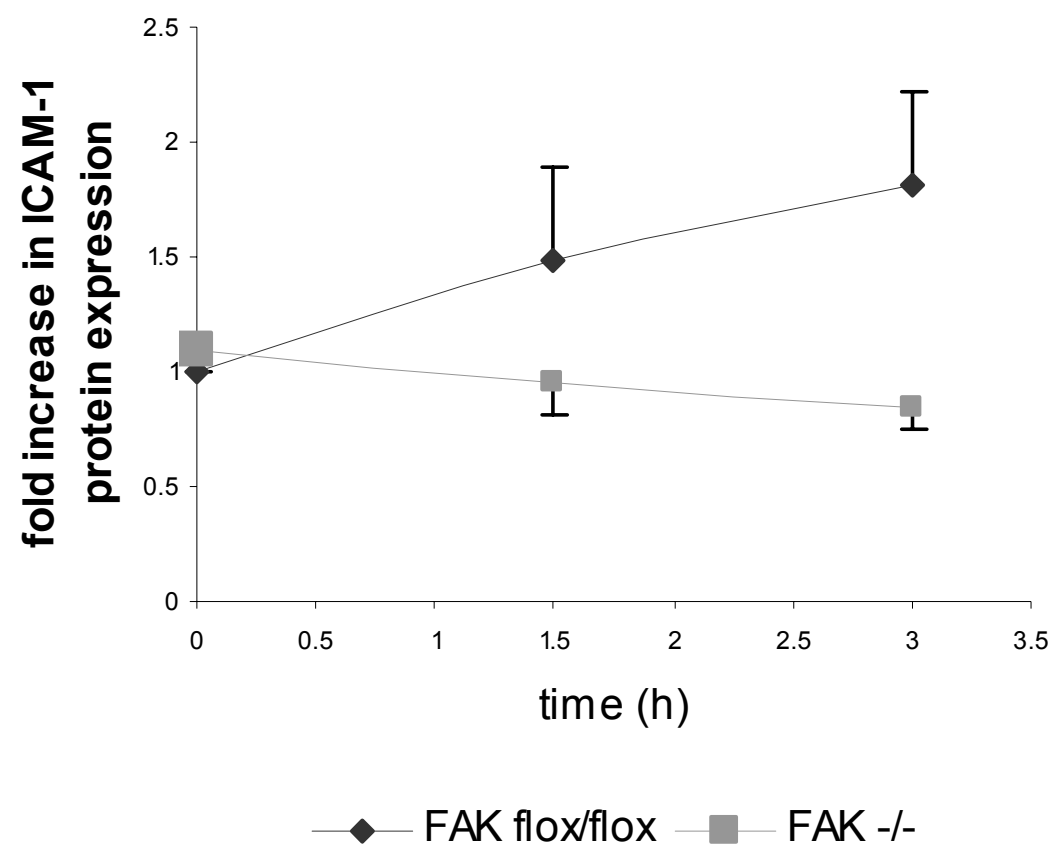

Figure 4C: Flow induced ICAM-1 expression. Cell were sheared for the indicated times and subjected to immunoblotting for ICAM-1. N=3, bars over time points represent standard errors. Values are normalized to total ERK protein levels.

Activated NF-kB is known to bind the shear stress response element (SSRE) in the ICAM promoter and enhance its protein expression. Both cell lines were sheared for $1.5 \mathrm{~h}$ and $3 \mathrm{~h}$ and 
ICAM-1 expression levels were compared. As seen in figure 4C, control cells respond with an 0.87 fold $\mathrm{SE} \pm 0.18$ increase in ICAM-1 expression after $3 \mathrm{~h}$, in contrast to FAK-/- cells, where ICAM-1 levels remain similar over time. Furthermore, preliminary data show that FAK-/- cells are not able to activate a SSRE driven lucifferase. These results show that NF-kB is transcriptional inactive in FAK-/- cells. Taken together, FAK is required for the transcriptional activation of NF-kB and subsequent expression of NF-kB target genes like ICAM-1, but is dispensable in flow-induced ERK activation.

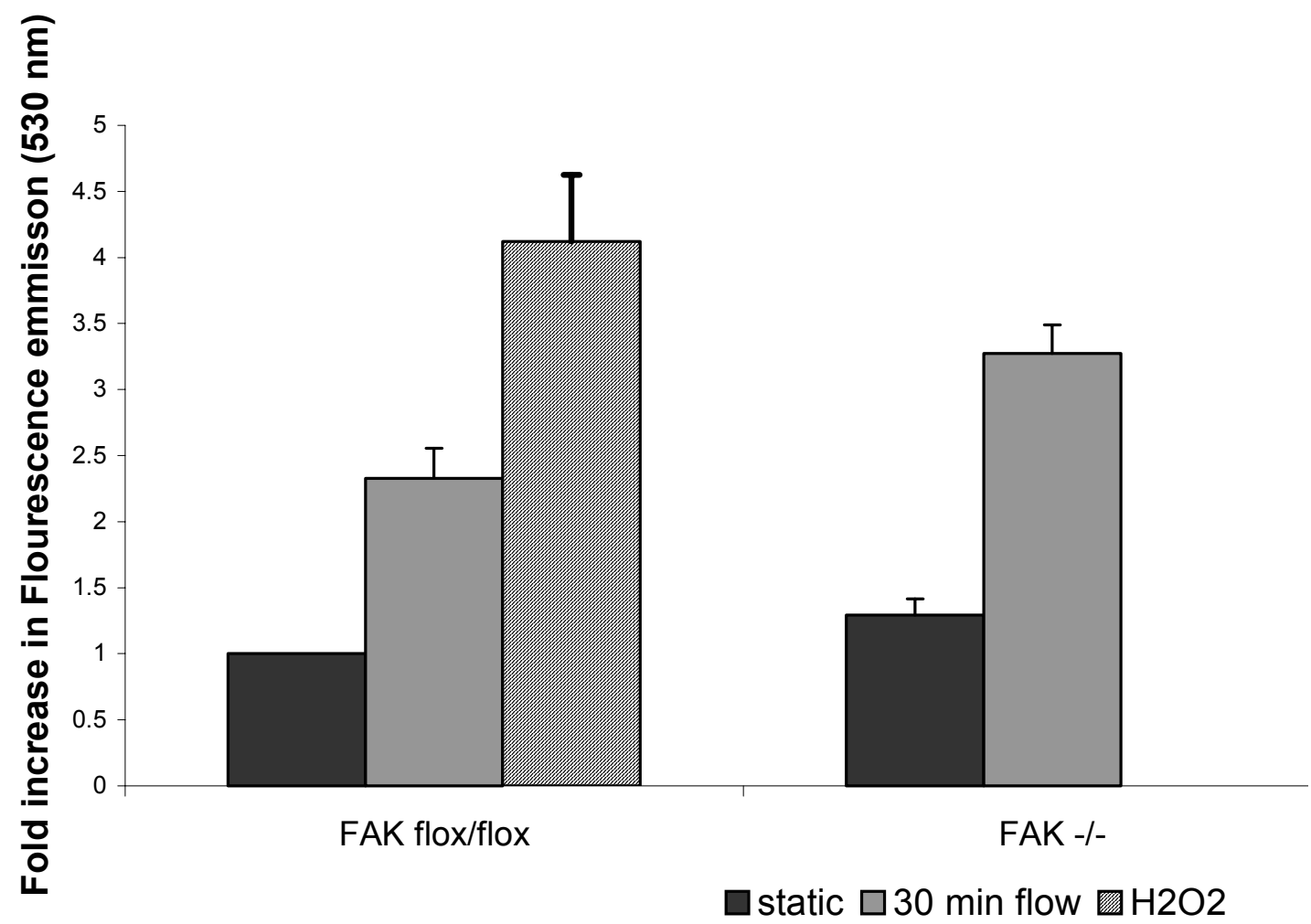

Fugre 5A: Flow induced production of Reactive oxygen species. Cell were treated as indicated and fluorescent was measured using a 96 well plate reader using a $530 \mathrm{~nm}$ emission \filter set. $\mathrm{N}=4$, Bars over time points represent standard errors. Fluorescent units were normalized towards total protein levels. 


\subsection{The Role of reactive oxygen species (ROS) in the activation of NF- $k B$ by flow}

NF-kB is activated by reactive oxygen species (ROS) and flow augments ROS production through a Rac-1, NADPH-oxidase dependent pathway. Thus, I was interested if ROS production is decreased in FAK-/- cells. DCFH-DA, a redox-sensitive compound that becomes fluorescent after oxidation, was used to assay the intracellular redox state. Prior to shearing, cells were incubated with DCFH for $30 \mathrm{~min}$ and then sheared in medium containing DCFH-DA. Subsequently, fluorescence of the lysate was determined using a fluorescent plate reader (emission filter ca. 530nm). As positive control I included $\mathrm{H}_{2} \mathrm{O}_{2}-(0.6 \mu \mathrm{M})$ stimulation of FAK flox/flox, which should induce ROS, as $\mathrm{H}_{2} \mathrm{O}_{2}$ is a known ROS inducer. Although FAK -/- cells generate slightly more ROS under static conditions the increase after flow was similar (Figure 5A). To rule out the possibility that FAK-/- cells are not able to respond towards augmented ROS levels, I treated cells with $0.6 \mu \mathrm{M} \mathrm{H}_{2} \mathrm{O}_{2}$ for 30 min and assayed p65 phosphorylation. Figure $5 \mathrm{~B}$ shows that both cell lines activate p65 to a similar extent.

In summary, these data imply that FAK-/- cells are able to produce and respond towards reactive oxygen species properly. Further, I conclude that FAK might to be required for a ROS independent step in NF-kB activation by flow. 


\section{DB: p65p}

\section{IB: tp65}

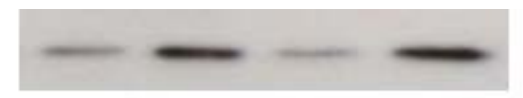

$\begin{array}{ccccc}\text { Time (min) } & 0 & 30 & 0 & 30 \\ & \text { FAK flox/flox } & & \text { FAK-/- }\end{array}$

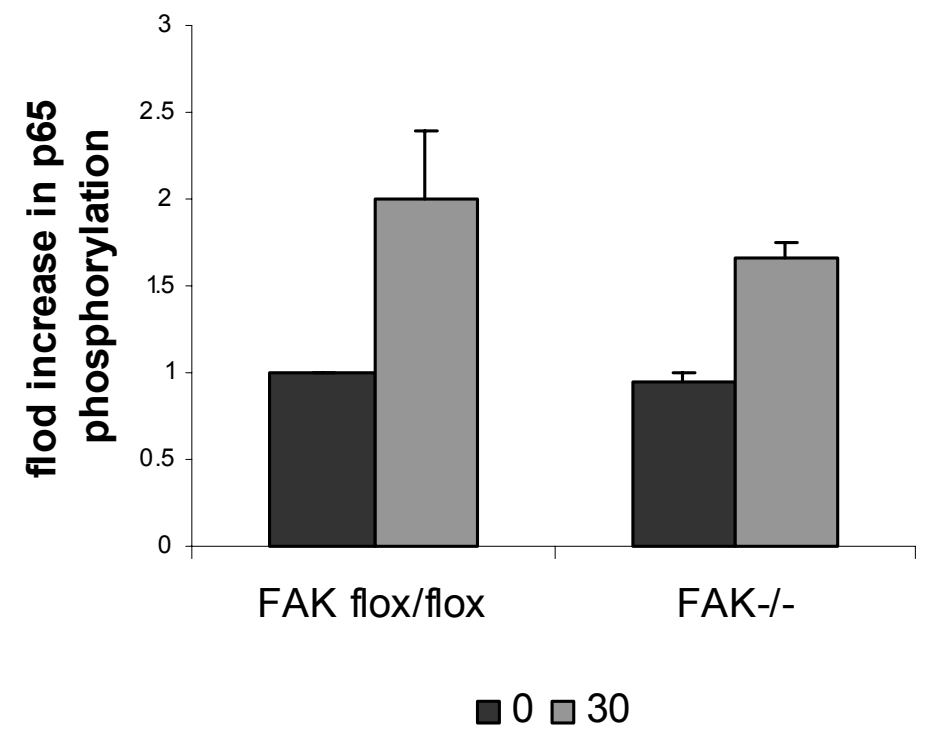

Figure 5B: ROS stimulated p65 phosphorylation. Cells were treated with with $0.6 \mu \mathrm{M} \mathrm{H}_{2} \mathrm{O}_{2}$ for 30 min and assayed p65 phosphorylation. $\mathrm{N}=3$, Bars over time points represent standard errors. Values are normalized to total p65 protein levels. 


\section{Discussion and future directions}

This study addressed the role of FAK in shear-induced signaling. By deriving a FAK-/endothelial cell line model, I established a useful tool to study FAK function in shear-induced signaling. I could not observe any major differences either in flow induced MAPK activation and ROS production nor in NF-kB response towards ROS in general. Interestingly, I showed that FAK is required for the activation of NF-kB by flow, most likely through a step distinct from ROS production. Subsequently, I observed that FAK ablation inhibits NF-kB-driven ICAM-1 protein expression, underlining the potential in vivo relevance as NF-kB and ICAM-1 are closely linked to the development of atherosclerosis.

I isolated FAK flox/flox conditional MAEC which maintain EC-specific characteristics in culture such as VE-cadherin expression and proper intercellular junctions, both essential for mechanotransduction events (Figure 1). However by deleting FAK in conditional FAK fl/fl MAEC I was able to fully inhibited FAK protein expression (Figure2). This FAK knock out cell model is superior to others to study protein function. Gene knock out is much more efficient than gene silencing with siRNA, because the gene of interest is completely deleted instead of having just reduced protein translation. In addition, it is more informative compared to dominant negative mutants (such as Y397F or FRNK) which might have off target effects and allow only limited conclusions about protein function.

In previous experiments, FAK-/- fibroblasts were reported to have augmented FA formation and Pyk2 protein expression $[40,42,71]$. As increased adhesion size might influence mechanotransduction events, I was curious if MAEC FAK-/- cells show more abundant adhesions. I stained for paxillin, another FAK independent FA marker and saw no differences in adhesion abundance between both cells, although this experiment did not address differences in FA dynamics which were reported by others $[42,73]$. As Pyk2, a FAK related protein tyrosine 
kinase, was shown to partially compensate for FAK function [71] I compared Pyk2 protein levels between control and knock out cells and did not find any difference (Figure 2). This difference between my findings and earlier studies might be due to different cell types and knock out approaches, used by different investigators.

Next, I looked for MAPK and NF-kB activation by flow. Both are activated by flow through well studied signaling pathways, and were shown to be integrin dependent $[24,27]$ and therefore potentially FAK dependent.

Erk activation by flow

Erk activation by flow is due to Ras GTPase-mediated activation of MAPKKK (Raf-1), the most upstream kinase in the conserved MAPK activation cascade. Berk et al [74] described 3 critical steps necessary for Erk activation: Raf-1 recruitment to the membrane via GTP-bound Ras, Raf1 tyrosine phosphorylation by src and serine/threonine phophorylation by PKCs. In addition, an earlier study using dominant negative mutants, suggested upstream involvement of FAK in this pathway, via FAK-mediated recruitment of Grb2/SOS-Ras GEF [24].

Erk was shown to be transiently activated by flow with a peak between 5 and 10 min [75]. I observed a similar activation pattern in control and knock out cells by assaying phosphorylation of Thr202 and Tyr204. I concluded that FAK does not play an essential role in Erk activation. Therefore, it seems likely that an integrin- and FAK- independent pathway such as emphasized by Osawa [76] mediates Erk activation by flow. Osawa showed that flow- induced PECAM-1 phosphorylation and subsequent recruitment of SHP2 phosphatase are essential for Erk activation. Therefore my experiment showed that both cell lines response in a proper way towards shear stress stimulation. 


\section{$N F-k B$ activation by flow}

Next I addressed the NF-kB pathway, which was shown to be activated by flow in an integrinand Rac-dependent manner [27]. Serine536 in the carboxy-terminal transactivation domain of Rel-A (p65) can be phosphorylated by an active IKK complex or ribosomal subunit kinase-1 and is critical for recruiting TATA binding proteins, transcription-factor II or cofactors to $\mathrm{kB}$ enhancer sites in different promoters [77]. Interestingly, my data showed that NF-kB is not phosphorylated in FAK -/- cells after flow (Figure 4B). Additionally, I could show that NF-kB is transcriptionally inactive and is unable to increase ICAM-1 protein expression in response to flow. (Figure 4C). Thus, FAK is required for the transcriptional activation of NF-kB and target gene expression by flow. Currently, I am working on the reconstitution of wild type FAK in FAK-/- cells which should rescue NF-kB activation. This experiment is required to exclude the possibility that other events such as viral infections or knock out side affects are responsible for the lack of NF-kB activity.

To explain the lack of NF-kB activation in FAK-/- cells, I considered that shear stress activates the IKK complex through a NADPH oxidase-dependent production of ROS [78]. Since active Rac actives the NADPH oxidase complex [50] and FAK is able to activate Rac GTPases [35], I was curious if FAK is required for NADPH-oxidase activation and production of ROS in response to shear. 
$R O S$

As shown in figure 5, FAK -/- cells produce similar amounts of ROS compared to control cells. To exclude the possibility that FAK knockout affects the ability of MAEC to activate NF-kB by ROS, I treated both cell lines with $\mathrm{H}_{2} \mathrm{O}_{2}$ for 30 min and could not find differences. This argues that FAK -/- cells exhibit proper IKK function, although this evidence has the limitation that the $\mathrm{H}_{2} \mathrm{O}_{2}$ stimulus was unphysiological high. Taken together these data argue that FAK is neither involved in flow induced ROS production nor in oxidant-mediated activation of NF-kB. Hence, it seems that FAK is required in a parallel or sequential signaling cascade to activate NF-kB by flow.

One possibility is that Ser536 phosphorylation by shear is facilitated by RSK1. RSK1 is reported to be activated by ROS, PI3K and MAPK cascade members that are known to be shear responsive (see above). Future experiments using dominant negative RSK1 mutants or specific inhibitors might test this hypothesis. Alternatively, IKK localization into adhesion sites could be required for NF-kB activation by flow. Orr et al [30] showed that flow-activated IKKs colocalized with $\beta 1$ integrins, whereas $\mathrm{TNF} \alpha$ treatment resulted in diffuse nuclear and cytoplasmic staining. If IKK recruitment to adhesion sites was via FAK, it might facilitate NF-kB activation by flow but not affect the response to added $\mathrm{H}_{2} \mathrm{O}_{2}$. Thereby FAK scaffolding function could be a key. So far, no conclusive evidence exists that integrated FAK into a NF-kB activation pathway by flow.

Future experiments could directly approach FAK function by using FAK mutants. An advantage of the knock out model is that it allows the reconstitution of site specific mutants which might help to identify potential downstream interacting partners. 
Clinical relevance

Finally I would like to emphasis a potential role of FAK in the development of atherosclerosis. In this study I showed that FAK in required for the NF-kB driven ICAM-1 expression. ICAM-1 is found to be overexpressed in atheroprone sites in the vasculature $[79,80]$ and mediates the firm arrest of leucocytes via their $\beta 2$ integrins to the activated endothelium. Additionally, high concentrations of soluble ICAM-1 is a positive predictor for future fatal and non fatal coronary artery disease events such as myocardial infarction [81]. Furthermore, activated NF-kB drives the expression of other pro-inflammatory markers such as monocyte chemoattractant protein-1 or VCAM-1, players in the development of atherosclerosis. Although it is far away from current experiments, future studies might address if FAK ablation (in the mouse model) or FAK inhibition by interfering drugs (clinical study) might decrease atherosclerosis and might have clinical benefits. 


\section{$\underline{\text { 5. References }}$}

1. Orr, A.W., et al., Mechanisms of mechanotransduction. Dev Cell, 2006. 10(1): p. $11-20$.

2. Lehoux, S., Y. Castier, and A. Tedgui, Molecular mechanisms of the vascular responses to haemodynamic forces. J Intern Med, 2006. 259(4): p. 381-92.

3. $\quad$ Reneman, R.S., T. Arts, and A.P. Hoeks, Wall Shear Stress - an Important Determinant of Endothelial Cell Function and Structure - in the Arterial System in vivo. Discrepancies with Theory. J Vasc Res, 2006. 43(3): p. 251-69.

4. Malek, A.M., S.L. Alper, and S. Izumo, Hemodynamic shear stress and its role in atherosclerosis. Jama, 1999. 282(21): p. 2035-42.

5. Cunningham, K.S. and A.I. Gotlieb, The role of shear stress in the pathogenesis of atherosclerosis. Lab Invest, 2005. 85(1): p. 9-23.

6. Dai, G., et al., Distinct endothelial phenotypes evoked by arterial waveforms derived from atherosclerosis-susceptible and -resistant regions of human vasculature. Proc Natl Acad Sci U S A, 2004. 101(41): p. 14871-6.

7. Cheng, C., et al., Atherosclerotic lesion size and vulnerability are determined by patterns of fluid shear stress. Circulation, 2006. 113(23): p. 2744-53.

8. Davies, P.F., Flow-mediated endothelial mechanotransduction. Physiol Rev, 1995. 75(3): p. 519-60.

9. Tzima, E., et al., Localized cdc42 activation, detected using a novel assay, mediates microtubule organizing center positioning in endothelial cells in response to fluid shear stress. J Biol Chem, 2003. 278(33): p. 31020-3.

10. McCue, S., et al., Shear stress regulates forward and reverse planar cell polarity of vascular endothelium in vivo and in vitro. Circ Res, 2006. 98(7): p. 939-46.

11. Li, Y.S., J.H. Haga, and S. Chien, Molecular basis of the effects of shear stress on vascular endothelial cells. J Biomech, 2005. 38(10): p. 1949-71.

12. Haidekker, M.A., N. L'Heureux, and J.A. Frangos, Fluid shear stress increases membrane fluidity in endothelial cells: a study with DCVJ fluorescence. Am J Physiol Heart Circ Physiol, 2000. 278(4): p. H1401-6.

13. Butler, P.J., et al., Shear stress induces a time- and position-dependent increase in endothelial cell membrane fluidity. Am J Physiol Cell Physiol, 2001. 280(4): p. C962-9.

14. Chien, S., Molecular basis of rheological modulation of endothelial functions: importance of stress direction. Biorheology, 2006. 43(2): p. 95-116.

15. Yu, J., et al., Direct evidence for the role of caveolin-1 and caveolae in mechanotransduction and remodeling of blood vessels. J Clin Invest, 2006. 116(5): p. 1284-91.

16. Tzima, E., et al., A mechanosensory complex that mediates the endothelial cell response to fluid shear stress. Nature, 2005. 437(7057): p. 426-31.

17. Katsumi, A., et al., Integrins in mechanotransduction. J Biol Chem, 2004. 279(13): p. 12001-4.

18. Hynes, R.O., Integrins: versatility, modulation, and signaling in cell adhesion. Cell, 1992. 69(1): p. 11-25. 
19. Schwartz, M.A. and R.K. Assoian, Integrins and cell proliferation: regulation of cyclin-dependent kinases via cytoplasmic signaling pathways. J Cell Sci, 2001. 114(Pt 14): p. 2553-60.

20. Mitra, S.K. and D.D. Schlaepfer, Integrin-regulated FAK-Src signaling in normal and cancer cells. Curr Opin Cell Biol, 2006. 18(5): p. 516-23.

21. Schwartz, M.A., Integrin signaling revisited. Trends Cell Biol, 2001. 11(12): p. 466-70.

22. Brakebusch, C. and R. Fassler, The integrin-actin connection, an eternal love affair. Embo J, 2003. 22(10): p. 2324-33.

23. Liu, S., D.A. Calderwood, and M.H. Ginsberg, Integrin cytoplasmic domainbinding proteins. J Cell Sci, 2000. 113 ( Pt 20): p. 3563-71.

24. Li, S., et al., Fluid shear stress activation of focal adhesion kinase. Linking to mitogen-activated protein kinases. J Biol Chem, 1997. 272(48): p. 30455-62.

25. Jalali, S., et al., Integrin-mediated mechanotransduction requires its dynamic interaction with specific extracellular matrix (ECM) ligands. Proc Natl Acad Sci U S A, 2001. 98(3): p. 1042-6.

26. Tzima, E., et al., Activation of integrins in endothelial cells by fluid shear stress mediates Rho-dependent cytoskeletal alignment. Embo J, 2001. 20(17): p. 463947.

27. Tzima, E., et al., Activation of Racl by shear stress in endothelial cells mediates both cytoskeletal reorganization and effects on gene expression. Embo J, 2002. 21(24): p. 6791-800.

28. Liu, Y., et al., Shear stress activation of SREBP1 in endothelial cells is mediated by integrins. Arterioscler Thromb Vasc Biol, 2002. 22(1): p. 76-81.

29. Urbich, C., et al., Laminar shear stress upregulates integrin expression: role in endothelial cell adhesion and apoptosis. Circ Res, 2000. 87(8): p. 683-9.

30. Orr, A.W., et al., The subendothelial extracellular matrix modulates NF-kappaB activation by flow: a potential role in atherosclerosis. J Cell Biol, 2005. 169(1): p. 191-202.

31. Orr, A.W., et al., Matrix-specific Suppression of Integrin Activation in Shear Stress Signaling. Mol Biol Cell, 2006. 17(11): p. 4686-4697.

32. Zaidel-Bar, R., et al., Early molecular events in the assembly of matrix adhesions at the leading edge of migrating cells. J Cell Sci, 2003. 116(Pt 22): p. 4605-13.

33. Parsons, J.T., Focal adhesion kinase: the first ten years. J Cell Sci, 2003. 116(Pt 8): p. 1409-16.

34. Schaller, M.D., Biochemical signals and biological responses elicited by the focal adhesion kinase. Biochim Biophys Acta, 2001. 1540(1): p. 1-21.

35. Parsons, J.T., et al., Focal adhesion kinase: a regulator of focal adhesion dynamics and cell movement. Oncogene, 2000. 19(49): p. 5606-13.

36. Braren, R., et al., Endothelial FAK is essential for vascular network stability, cell survival, and lamellipodial formation. J Cell Biol, 2006. 172(1): p. 151-62.

37. Shen, T.L., et al., Conditional knockout of focal adhesion kinase in endothelial cells reveals its role in angiogenesis and vascular development in late embryogenesis. J Cell Biol, 2005. 169(6): p. 941-52. 
38. Quadri, S.K. and J. Bhattacharya, Resealing of endothelial junctions by focal adhesion kinase. Am J Physiol Lung Cell Mol Physiol, 2006.

39. Quadri, S.K., et al., Endothelial barrier strengthening by activation of focal adhesion kinase. J Biol Chem, 2003. 278(15): p. 13342-9.

40. Ilic, D., et al., Reduced cell motility and enhanced focal adhesion contact formation in cells from FAK-deficient mice. Nature, 1995. 377(6549): p. 539-44.

41. Ilic, D., et al., FAK promotes organization of fibronectin matrix and fibrillar adhesions. J Cell Sci, 2004. 117(Pt 2): p. 177-87.

42. Ren, X.D., et al., Focal adhesion kinase suppresses Rho activity to promote focal adhesion turnover. J Cell Sci, 2000. 113 ( Pt 20): p. 3673-8.

43. Koshida, R., et al., Role of focal adhesion kinase in flow-induced dilation of coronary arterioles. Arterioscler Thromb Vasc Biol, 2005. 25(12): p. 2548-53.

44. Hayden, M.S. and S. Ghosh, Signaling to NF-kappaB. Genes Dev, 2004. 18(18): p. 2195-224.

45. Collins, T. and M.I. Cybulsky, NF-kappaB: pivotal mediator or innocent bystander in atherogenesis? J Clin Invest, 2001. 107(3): p. 255-64.

46. Chen, Z.J., Ubiquitin signalling in the NF-kappaB pathway. Nat Cell Biol, 2005. 7(8): p. 758-65.

47. Libby, P., P.M. Ridker, and A. Maseri, Inflammation and atherosclerosis. Circulation, 2002. 105(9): p. 1135-43.

48. Hajra, L., et al., The NF-kappa B signal transduction pathway in aortic endothelial cells is primed for activation in regions predisposed to atherosclerotic lesion formation. Proc Natl Acad Sci U S A, 2000. 97(16): p. 9052-7.

49. Tzima, E., Role of small GTPases in endothelial cytoskeletal dynamics and the shear stress response. Circ Res, 2006. 98(2): p. 176-85.

50. Babior, B.M., NADPH oxidase. Curr Opin Immunol, 2004. 16(1): p. 42-7.

51. Schreck, R., P. Rieber, and P.A. Baeuerle, Reactive oxygen intermediates as apparently widely used messengers in the activation of the NF-kappa B transcription factor and HIV-1. Embo J, 1991. 10(8): p. 2247-58.

52. Yusuf, S., et al., Global burden of cardiovascular diseases: part I: general considerations, the epidemiologic transition, risk factors, and impact of urbanization. Circulation, 2001. 104(22): p. 2746-53.

53. Imakita, M., et al., Second nation-wide study of atherosclerosis in infants, children and young adults in Japan. Atherosclerosis, 2001. 155(2): p. 487-97.

54. VanderLaan, P.A., C.A. Reardon, and G.S. Getz, Site specificity of atherosclerosis: site-selective responses to atherosclerotic modulators. Arterioscler Thromb Vasc Biol, 2004. 24(1): p. 12-22.

55. Caro, C.G., J.M. Fitz-Gerald, and R.C. Schroter, Arterial wall shear and distribution of early atheroma in man. Nature, 1969. 223(5211): p. 1159-60.

56. Brooks, A.R., P.I. Lelkes, and G.M. Rubanyi, Gene expression profiling of human aortic endothelial cells exposed to disturbed flow and steady laminar flow. Physiol. Genom., 2002. 9: p. 27-41.

57. Dai, G., et al., Distinct endothelial phenotypes evoked by arterial waveforms derived from atherosclerosis-susceptible and-resistant regions of human vasculature. Proc Natl Acad Sci U S A, 2004. 
58. Keulenaer, G.W.D., et al., Oscillatory and steady laminar shear stress differentially affect human endothelial redox state: role of a superoxideproducing NADH oxidase. Circ. Res., 1998. 82: p. 1094-1101.

59. Mohan, S., N. Mohan, and E.A. Sprague, Differential activation of $N F-k B$ in human aortic endothelial cells conditioned to specific flow environments. Am. J. Physiol. (Cell Physiol.), 1997. 273: p. C572-C578.

60. Topper, J.N., et al., Identification of vascular endothelial genes differentially responsive to fluid mechanical stimuli: cyclooxygenase-2, manganese superoxide dismutase, and endothelial cell nitric oxide synthase are selectively up-regulated by steady laminar shear stress. Proc. Natl Acad. Sci USA, 1996. 93: p. 1041710422 .

61. Gimbrone, M.A., Jr., et al., Endothelial dysfunction, hemodynamic forces, and atherogenesis. Ann N Y Acad Sci, 2000. 902: p. 230-9; discussion 239-40.

62. Barry-Lane, P.A., et al., p47phox is required for atherosclerotic lesion progression in ApoE(-/-) mice. J Clin Invest, 2001. 108(10): p. 1513-22.

63. Himburg, H.A., et al., Spatial comparison between wall shear stress measures and porcine arterial endothelial permeability. Am J Physiol Heart Circ Physiol, 2004. 286(5): p. H1916-22.

64. Srinivasan, S., et al., Modulation of PPARalpha expression and inflammatory interleukin-6 production by chronic glucose increases monocyte/endothelial adhesion. Arterioscler Thromb Vasc Biol, 2004. 24(5): p. 851-7.

65. Gilbert, S.F. and B.R. Migeon, D-valine as a selective agent for normal human and rodent epithelial cells in culture. Cell, 1975. 5(1): p. 11-7.

66. Beggs, H.E., et al., FAK deficiency in cells contributing to the basal lamina results in cortical abnormalities resembling congenital muscular dystrophies. Neuron, 2003. 40(3): p. 501-14.

67. Nerem, R.M., et al., The study of the influence of flow on vascular endothelial biology. Am J Med Sci, 1998. 316(3): p. 169-75.

68. Wang, H. and J.A. Joseph, Quantifying cellular oxidative stress by dichlorofluorescein assay using microplate reader. Free Radic Biol Med, 1999. 27(5-6): p. 612-6.

69. Lowry, O.H., et al., Protein measurement with the Folin phenol reagent. J Biol Chem, 1951. 193(1): p. 265-75.

70. Ichaso, N. and S.M. Dilworth, Cell transformation by the middle T-antigen of polyoma virus. Oncogene, 2001. 20(54): p. 7908-16.

71. Sieg, D.J., et al., Pyk2 and Src-family protein-tyrosine kinases compensate for the loss of FAK in fibronectin-stimulated signaling events but Pyk2 does not fully function to enhance FAK- cell migration. Embo J, 1998. 17(20): p. 5933-47.

72. Burridge, K. and M. Chrzanowska-Wodnicka, Focal adhesions, contractility, and signaling. Annu Rev Cell Dev Biol, 1996. 12: p. 463-518.

73. Webb, D.J., et al., FAK-Src signalling through paxillin, ERK and MLCK regulates adhesion disassembly. Nat Cell Biol, 2004. 6(2): p. 154-61.

74. Traub, O. and B.C. Berk, Laminar shear stress: mechanisms by which endothelial cells transduce an atheroprotective force. Arterioscler Thromb Vasc Biol, 1998. 18(5): p. 677-85. 
75. Tseng, H., T.E. Peterson, and B.C. Berk, Fluid shear stress stimulates mitogenactivated protein kinase in endothelial cells. Circ Res, 1995. 77(5): p. 869-78.

76. Osawa, M., et al., Evidence for a role of platelet endothelial cell adhesion molecule-1 in endothelial cell mechanosignal transduction: is it a mechanoresponsive molecule? J Cell Biol, 2002. 158(4): p. 773-85.

77. Chen, L.F. and W.C. Greene, Shaping the nuclear action of NF-kappaB. Nat Rev Mol Cell Biol, 2004. 5(5): p. 392-401.

78. Mohan, S.N., et al., Low Shear Stress Preferentially Enhances IKK Activity Through Selective Sources Of Reactive Oxygen Species For Persistent Activation Of NF-k\{beta\} In Endothelial Cells. Am J Physiol Cell Physiol, 2006.

79. Libby, P., Inflammation in atherosclerosis. Nature, 2002. 420(6917): p. 868-74.

80. Nakashima, Y., et al., Upregulation of VCAM-1 and ICAM-1 at atherosclerosisprone sites on the endothelium in the ApoE-deficient mouse. Arterioscler Thromb Vasc Biol, 1998. 18(5): p. 842-51.

81. Blankenberg, S., S. Barbaux, and L. Tiret, Adhesion molecules and atherosclerosis. Atherosclerosis, 2003. 170(2): p. 191-203.

Book references:

82. Löffler, Georg: Basiswissen Biochemie mit Pathobiochemie. 5. Auflage, Springer Verlag Berlin, Heidelberg, 2003

83. Schmidt, Lang, Thews: Physiologie des Menschen, 28. Auflage, Springer Verlag Berlin, Heidelberg, 2000 